\title{
The influence of inter-particle friction and the intermediate stress ratio on soil response under generalised stress conditions
}

Dr Daniel Barreto ${ }^{1}$ (Corresponding author)

School of Engineering and the Built Environment

Edinburgh Napier University

Merchiston Campus

Edinburgh, EH10 5DT

Tel: $\quad+44(0) 1314552422$

Fax: $\quad+44(0) 1314552264$

e-mail: d.barreto@napier.ac.uk

Dr Catherine O'Sullivan

Department of Civil and Environmental Engineering

Imperial College London

South Kensington Campus

London, SW7 2AZ

Tel: $\quad+44(0) 2075946117$

Fax: $\quad+44(0) 2075945934$

e-mail: cath.osullivan@imperial.ac.uk

${ }^{1}$ Formerly Imperial College London 


\begin{abstract}
Previous research studies have used either physical experiments or discrete element method (DEM) simulations to explore, independently, the influence of the coefficient of inter-particle friction $(\mu)$ and the intermediate stress ratio $(b)$ on the behaviour of granular materials. DEM simulations and experiments using photoelasticity have shown that when an anisotropic stress condition is applied to a granular material, strong force chains or columns of contacting particles transmitting relatively large forces, form parallel to the major principal stress orientation. The combined effects of friction and the intermediate stress ratio upon the resistance of these strong force chains to collapse (buckling failure) are considered here using data from an extensive set of DEM simulations including triaxial and true triaxial compression tests. For all tests both $\mu$ and $b$ affected the macroand micro-scale response, however the mechanisms whereby the force chain stability was improved differ. While friction clearly enhances the inherent stability of the strong force chains, the intermediate stress ratio affects the contact density and distribution of orthogonal contacts that provide lateral support to the force chains.
\end{abstract}

Key words: DEM, true triaxial tests, fabric, intermediate stress ratio, interparticle friction 


\section{The influence of inter-particle friction and the intermediate stress ratio on soil response under generalised stress conditions}

\section{Introduction}

Granular materials respond to applied loads in a highly complex manner. One mechanical response feature unique to granular materials is the sensitivity of the response to the intermediate principal stress. This paper makes a contribution to fundamental understanding of granular material response by examining the combined effects of friction and the relative magnitude of the intermediate principal stress on the material response. This study involved an extensive set of three-dimensional discrete element method (DEM) simulations using periodic boundaries. The first series of test simulations were triaxial compression tests on samples with equal packing density but differing coefficients of friction, $\mu$. Then the analysis was extended to true triaxial test simulations where the samples were subjected to axial compression at a constant mean stress for a range of intermediate stress ratios between 0 and 1 . The study extends the earlier contributions of $\mathrm{Ng}[1]$ and Thornton [2].

The paper outlines the simulation approach and presents the macro-scale, overall mechanical load-deformation response. Quantitative analysis of the material fabric using the contact normal force orientations and distributions allowed observations on the fundamental mechanisms underlying the observed response to be made. The results indicate that the friction coefficient, $\mu$, influences the inherent stability of the strong force chains, while the intermediate stress ratio influences the lateral support provided to these force chains. 


\section{Simulation parameters and specimen generation}

The distinct element method (DEM) simulations presented here were performed on threedimensional poly-disperse assemblies of spheres. A Hertz-Mindlin contact model was used as in numerous prior DEM simulations [e.g. 3 - 4]. The Poisson's ratio (v) was 0.22, the shear modulus $(\mathrm{G})$ was $28.68 \mathrm{GPa}$ and the particle density $(\rho)$ was $2570 \mathrm{~kg} / \mathrm{m}^{3}$. The particle size distribution (PSD) was produced using seven different particle sizes, giving a mean particle diameter $\left(\mathrm{d}_{50}\right)$ of $1.1 \mathrm{~mm}$ and minimum $\left(\mathrm{d}_{\text {min }}\right)$ and maximum $\left(\mathrm{d}_{\max }\right)$ particle sizes of $1.005 \mathrm{~mm}$ and $1.425 \mathrm{~mm}$ respectively. This PSD was selected to match the properties of glass ballotini particles used in a complementary experimental study by Barreto [5]. The DEM code used in this study was a modified version of ELLIPSE3D [6] which is based upon the TRUBAL code developed by Cundall and Strack [7]. This code was modified in the current study, following previous updates by O'Sullivan [8] and Cui [9]. The simulations presented here were performed using periodic boundaries. Such boundaries are widely used in geotechnical engineering and details for their implementation can be found elsewhere $[2,10]$

The use of a periodic cell in a DEM simulation removes boundary effects and allows smaller numbers of particles to be considered than would be needed to get representative data in a physical experiment. However if the number of particles included in the periodic cell is too small, the results may not be representative of the general material behaviour. Consequently a parametric study was performed in order to select the optimum cell size. Simulations with 2000, 4000 and 8000 particles provided identical macro-scale results when the stress paths and the angles of shearing resistance were compared. However, quantification of the internal topology or fabric (using the fabric tensor as defined by Satake [11]) revealed differences of up to $31 \%$ between the assemblies with 2000 particles and the other two specimen sizes, indicating that the sample was too small for the topology to be statitiscally representative. The good agreement in the response of the specimens containing 4000 and 8000 spheres meant that simulations of 4000 particles 
were used to obtain valid results with reasonable simulation run times. These findings are not general as the minimum acceptable periodic cell size will depend on the particle size and shape distributions.

Isotropic specimens were created by initially generating a cloud of non-contacting spheres with random locations within a cubic periodic cell of $33.9 \mathrm{~mm} \times 33.9 \mathrm{~mm} \times 33.9$ $\mathrm{mm}$. Then the samples were compressed isotropically and monotonically to $200 \mathrm{kPa}$. By setting $\mu$ to be 0.0 during compression relatively dense samples with a void ratio (e) of 0.521 were created. Note that $\mathrm{e}$ is defined as the ratio between the volume of voids and the volume of solid particles. During consolidation e decreases, the number of interparticle contacts increases and prior to shearing, the particle assembly is percolating, i.e. there is a network of contacts with sufficient stability to transmit stress through the sample.

\section{Influence of inter-particle friction and the intermediate principal stress during triaxial and true-triaxial compression}

\subsection{Axi-symmetric (triaxial) compression}

Figure 1(a) illustrates the stress-strain response in terms of the deviatoric stress $\left(t=\left(\sigma_{1}^{\prime}\right.\right.$ -

$\left.\sigma_{3}^{\prime}\right) / 2$ ) for triaxial compression tests with various values of $\mu$. Note that $\sigma_{1}^{\prime}$ and $\sigma_{3}^{\prime}$ are the major and minor principal effective stresses, respectively. The angle of shearing resistance increases as $\mu$ increases, in line with the observation of Thornton [2] amongst others. The peak strength was mobilized at approximately the same value of deviatoric strain $(\cong 8.5 \%)$ for all friction values. Taking the variation of the gradient of the stressstrain response as a measure of the evolution of yielding, for low friction yielding occurs gradually and for high friction yielding occurs suddenly at relatively low strain levels, and there is a systematic evolution in the response as $\mu$ is varied. As illustrated in Figure 
1(b), the overall dilative volumetric response observed is consistent with the initially dense state of the assemblies. After a limited initial compression at strain levels lower than $0.20 \%$ the samples start to dilate at a constant rate (see Figure 1(c)). The magnitude of this initial compression is small, and it is dependant on $\mu$ with a larger amount of compression being associated with the higher values of $\mu$. During the subsequent dilation the rate of dilation increases with $\mu$.

The relationship between friction and stiffness, evident in Figure 1(a), can be quantiatively appreciated by considering the octahedral stiffness $G_{\text {oct }}$, defined as $\mathrm{G}_{\mathrm{oct}}=\Delta \mathrm{J} / \Delta \varepsilon_{\mathrm{d}}$, with :

$$
\begin{gathered}
J=\frac{1}{\sqrt{6}}\left[\left(\sigma_{1}^{\prime}-\sigma_{2}^{\prime}\right)^{2}+\left(\sigma_{2}^{\prime}-\sigma_{3}^{\prime}\right)^{2}+\left(\sigma_{1}^{\prime}-\sigma_{3}^{\prime}\right)^{2}\right]^{0.5} \\
\varepsilon_{d}=\frac{2}{\sqrt{6}}\left[\left(\varepsilon_{1}-\varepsilon_{2}\right)^{2}+\left(\varepsilon_{2}-\varepsilon_{3}\right)^{2}+\left(\varepsilon_{1}-\varepsilon_{3}\right)^{2}\right]^{0.5}
\end{gathered}
$$

where $\mathbf{J}$ is a principal stress invariant in terms of the major, intermediate and minor principal effective stresses $\left(\sigma^{\prime}{ }_{1}, \sigma_{2}^{\prime}\right.$ and $\left.\sigma_{3}^{\prime}\right), \varepsilon_{\mathrm{d}}$ is the deviatoric strain and $\varepsilon_{1}, \varepsilon_{2}$ and $\varepsilon_{3}$ are the major, intermediate and minor principal strain values, respectively. The data presented in Figure 2(a) indicate that the sensitivity of $\mathrm{G}_{\text {oct }}$ to $\mu$ decreases with increasing deviatoric strain. The non-linear nature of the relationship between $\mu$ and $G_{\text {oct }}$ is clear from Figure 2(b) where the variation of $\mathrm{G}_{\mathrm{oct}}$ at different strain levels with $\mu$ are presented.

Referring to findings from prior research a conceptual fundamental mechanism explaining macro-scale response observed in Figures 1 and 2 can be developed. Bulk instabilities are induced by the continual collapse of old, and formation of new force chains [12] where force chains are defined as particle assemblies carrying the majority of the load (i.e. particles carrying forces greater than the average magnitude of the contact force). These force chains are supported laterally by a complimentary weak network of particles [13]. Prior to collapse force chains accumulate stored potential energy and this 
process continues until the force chains reach their peak load, after which buckling occurs and the force chains experience a loss in load-carrying capacity [14]. Furthermore, Tordesillas \& Muthuswamy [15] showed that force chain buckling can be elastic or plastic depending on the state of the contact points. Hence, failure is associated with buckling of the strong force chains and $\mu$ provides a resistance to buckling. Referring to Figure 1(a), when $\mu$ is low, inter-particle sliding (yielding of contacts in the shear direction) will occur at a lower strain level than when $\mu$ is higher. High values of $\mu$ provide a more inherently stable structure resulting in almost simultaneous buckling of the strong force chains. On the other hand, at low friction some of the strong force chains can yield while others have not reached the limiting value for sliding, hence yielding is more gradual. Earlier research [14] has showed that void spaces are created between the particles in the buckling chain and their laterally supporting neighbours and that the particle movements during buckling generate an inherently dilatant material response, as confirmed in Figures 1(b) and (c). The data presented here also show that at high values of $\mu$ collapse (buckling) is faster. It may then be possible to define collapse as the point of initiation of a constant rate of dilation. Figure 1(c) shows that this point is friction-dependant.

Figure 3(a) shows the evolution of the coordination number $\mathrm{Z}$ for different values of $\mu ; \mathrm{Z}$ is given by $2 \mathrm{~N}_{c} / \mathrm{N}_{\mathrm{p}}$ where $\mathrm{N}_{\mathrm{c}}$ and $\mathrm{N}_{\mathrm{p}}$ are the number of contacts and particles in the specimen respectively. Note that the number of contacts is multiplied by 2 because each contact is shared by two particles and this definition was used by Thornton [2]. All simulations have an initial value of $\mathrm{Z}=6.1$ and $\mathrm{Z}$ reduces as shearing progresses until a constant or "critical" coordination number, $Z_{\text {crit, }}$ is reached. The value of $Z_{\text {crit }}$ reduces with increasing $\mu$ confirming that friction adds significant stability to the force chain network. This stability associated with the higher $\mu$ values allows the stress to be transmitted using a smaller number of contacts and referring to Figure 1(a), the resultant mobilised shear strength is also higher. This ability to transmit stress with a smaller 
number of engaged contacts probably explains the increased dilative volumetric strain observed for the simulations with a higher $\mu$ value. The level of strain required to attain $\mathrm{Z}_{\text {crit }}$ increases with $\mu$.

Only a limited amount of information is provided by scalar measures of the material fabric such as Z. A deeper understanding can be gained using orientation data that can be quantified using the fabric tensor, defined by Satake [11] as:

$$
\phi_{i j}=\frac{1}{N_{c}} \sum_{k=1}^{N_{c}} n_{i}^{k} n_{j}^{k}
$$

where $N_{c}$ is the total number of contacts and $n_{i}^{k}$ denotes the unit contact normal for the $k$ th contact. Thornton [2] demonstrated the merits of considering the evolution of the deviatoric fabric $\Phi_{\mathrm{d}}\left(=\Phi_{1}-\Phi_{3}\right)$, where $\Phi_{1}$ and $\Phi_{3}$ are the major and minor fabric components, i.e. the maximum and minimum eigenvalues of $\Phi_{\mathrm{ij}}$. Figure 3(b) illustrates the evolution of $\Phi_{\mathrm{d}}$ for different friction values. There is clearly a correlation between a higher mobilised shear resistance and a higher deviatoric fabric. When friction is higher the strong force chains are less prone to buckling, i.e. they do not rely as much on an orthogonal force network to prop them up and facilitate transmission of the deviator stress. It is easy to imagine that when the friction is lower, the force chains buckle and the resulting lateral movement of the particles generates new contacts with adjacent particles, thus developing an orthogonal contact system. Figure 3(c) illustrates the evolution of the product of $\mathrm{Z}$ and $\Phi_{\mathrm{d}}$ for different $\mu$ values as shearing progresses. For $\mu>0.20 Z \Phi_{\mathrm{d}}$ is almost independent of $\mu$, implying that the number of particles participating in the strong force chains is approximately constant.

A number of researchers [16 - 18], have put forward functional relationships between $\mathrm{Z}$ and e. The continued dilative response observed here (Figure 1(b)) at a constant coordination number (Figure 3(a)) indicates that $\mathrm{Z}$ and e are not related via a simple oneone function. In Figure 3(d) a plot of $\mathrm{Z}$ against e and contours illustrating the value of 
the deviatoric fabric, are overlain on this data. For each test, once a critical coordination number is attained, there is a monotonic increase in e accompanied by an increase in $\Phi_{\mathrm{d}}$. Therefore the emergence of a constant coordination number is not an indicator of a constant or steady state fabric, rather as the deformation continues the contact network topology is evolving and the rate at which contacts are being lost in the direction of the minor principal fabric is approximately equal to the rate of gain of contacts in the direction of the major principal fabric. Consistently for each friction value, as shearing progresses the orthogonal support mobilized by the strong force chain network diminishes. However, while rational, this interpretation is itself a simplification of reality; clearly as shearing continues the $\Phi_{\mathrm{d}}$ contours are further apart, and for the higher friction values the increse in void ratio occurs at an almost constant $\Phi_{\mathrm{d}}$ value.

Various authors $[2,13]$ have observed_observed that in granular assemblies there are-are a number of particles that do not contribute to the load-carrying capacity. Based on this, Thornton [2] defined the mechanical coordination number $\left(\mathrm{Z}_{\mathrm{m}}\right)$ as:

$$
\mathrm{Z}_{\mathrm{m}}=\frac{2 \mathrm{~N}_{\mathrm{c}}-\mathrm{N}_{1}}{\mathrm{~N}_{\mathrm{p}}-\mathrm{N}_{0}-\mathrm{N}_{1}} \quad[\mathrm{X}]
$$

$\underline{\text { where }} N_{\underline{l}}$ and $N_{0}$ are the number of particles with 1 and 0 contacts, respectively, and $N_{\underline{C}}$ and $N_{p}$ are defined as before. However, when Figure 3(a) is compared to Figure 4(a) it seems apparent that the particular definition used for the coordination number does not affect the conclusions derived in this paper. In contrast, the evolution of the number of "rattlers" (particles with no load-capacity, $N_{\underline{l}}+N_{0}$ ) as illustrated in Figure X(b) showsthat as $m$ increases the number of rattlers also increases. It can be then confirmed that at high friction the force chains are do not rely as much on the orthogonal force network to prop them up. Hence, friction adds inherent stability to the force chains.

Three-dimensional work by Thornton and Anthony [19] defined "strong" contacts to be those carrying a normal force, $\mathrm{N}$, equal or greater than the average normal contact force $\langle N\rangle$. A similar definition was proposed in the earlier two-dimensional work of Radjai et 
al [13]. In this paper we consider a similar decomposition to study the evolution of the strong (s) and weak (w) fabrics, $\Phi_{\mathrm{ij}}^{\mathrm{s}}$ and $\Phi_{\mathrm{ij}}{ }^{\mathrm{w}}$, with $\Phi_{\mathrm{ij}}{ }^{\mathrm{s}}$ and $\Phi_{\mathrm{ij}}{ }^{\mathrm{w}}$ being calculated by applying Equation 3 only to contacts where $\mathrm{N} \geq\langle\mathrm{N}\rangle$ and $\mathrm{N}<\langle\mathrm{N}\rangle$ respectively. This proposition is based on evidence by Radjai et al [13] that the weak force network has its largest value when the mean force is used to separate the two networks. The evolution of $\Phi_{1}{ }^{\mathrm{s}}$ and $\Phi_{3}{ }^{\mathrm{s}}$ (strong fabric) is presented in Figure 4(a), while the evolution of $\Phi_{1}{ }^{\mathrm{w}}$ and $\Phi_{3}{ }^{\mathrm{w}}$ (weak fabric) is presented Figure 4(b) for the various $\mu$ values considered.

Referring to Figure 4(a), it is clear that the strong force network becomes highly anisotropic reaching maximum values of $0.521<\Phi_{1}{ }^{\mathrm{s}}<0.547$ (and $0.28<\Phi_{\mathrm{d}}{ }^{\mathrm{s}}<0.32$ ). The $\Phi_{1}{ }^{\mathrm{s}}$ values correlate well with the t data (Figure 1(a)); there is a "stiffer" response as $\mu$ increases and the peak fabric value also occurs at a similar strain level for all the simulations. A limiting value of principal fabric (or an envelope) seems to occur when $\mu$ is larger than 0.14 . However, the fabric tensor is normalised by the number of contacts, so misleading interpretations must be avoided, because while a similar degree of anisotropy is developed in the major principal direction, this magnitude of anisotropy is produced by an increasingly smaller number of contacts as $\mu$ increases. The weak force network $\left(\Phi_{i}{ }^{\mathrm{w}}\right)$ in Figure 4(b) exhibits a much smaller variation $\left(0.333<\Phi_{1}{ }^{\mathrm{w}}<0.372\right.$ compared to 0.333 $\left.<\Phi_{1}{ }^{\mathrm{S}}<0.547\right)$, remaining almost isotropic for the entire duration of shearing. This suggests that friction increases the stability of the strong force chains while the stability of the weak (and laterally supporting) network remains largely unaffected by $\mu$. The response observed in Figure 4(a) demonstrates that at low to moderate strain levels (i.e. $\varepsilon_{d}$ $\leq 4 \%$ ) a stronger principal fabric develops at a faster rate as $\mu$ increases. Such phenomenon cannot be observed in Figure 4(b) where the overall change of $\Phi_{1}{ }^{\mathrm{w}}$ is also much more limited. Consequently, it can be stated that friction only contributes to the stability of the strong force network. It is however (at least intuitively) expected that $\mu$ will affect the stability of both the strong and weak contact forces. However the results 
presented here suggest that for this packing density and stress level the increase in stability of the weak force network has no significant effect on the macro-scale behaviour because it only carries a limited amount of the overall load. It is expected that a higher stress levels the contribution of the weak force network will become increasingly significant and its enhanced stability will have an evident effect at both micro- and macro-scale levels.

The eigenvectors of $\Phi_{\mathrm{ij}}$ were used to determine the orientation of the strong and weak fabric. Taking $\beta^{\sigma}$ and $\beta^{\Phi}$ as the orientation of $\sigma_{1}$ and $\Phi_{1}$ with respect to the horizontal direction, respectively, for all simulations $\beta^{\sigma}=90^{\circ}$ and $\beta^{\Phi}{ }_{\mathrm{s}} \approx 85.5^{\circ}$. As the weak fabric is approximately isotropic, calculation of a principal orientation is not meaningful. This occurs because when there is an almost isotropic fabric there is no significant bias in a particular direction. As a result the eigenvectors can be significantly affected by very small changes in the fabric tensor, therefore producing random values of $\beta^{\Phi}{ }_{w}$. Once again, at a higher stress level it would be expected that the weak force would be orientated orthogonally to the direction of the major principal stress direction if its contribution to the stability becomes more significant, but this is not the case for the results presented here as discussed above.

Visual inspection of the contact force network (Figure 5) for two values of $\mu$ confirms this interpretation of the fabriethat the strong force network changes significantly, while the weak force network remains largely unaffected by $\mu$. This confirms the interpretation provided regarding the quantitative data in Figure 4. Strong contact forces (where $\mathrm{N} \geq\langle\mathrm{N}\rangle$ ) are plotted in black while weak contact forces are shown in gray and the line thickness is proportional to the magnitude of the normal force. Figure 5 (a) with $\mu=0.01$ shows a greater number of strong forces than Figure $5(b)$ with $\mu=0.5$. Interestingly the weak contact force network is very similar for both values of friction. Figures 1, 3, 4 and 5 demonstrate that with high friction a higher shear strength is mobilised with a smaller 
number of strong forces. Therefore we can conclude that the main role of $\mu$ is to provide an inherent resistance to strong force chain buckling by reducing the risk of lateral contact sliding in the force chain.

\subsection{True triaxial compression (Constant $b$ tests)}

The influence of $\mu$ on the shear strength of granular materials has been studied experimentally [i.e. 20 - 21] and numerically using DEM by various researchers [i.e. 2, 4, 22]. However, we know of no prior research considering the effect of friction on true triaxial response. This paper therefore extends the earlier DEM studies of true triaxial response by $\mathrm{Ng}[1,23]$ and Thornton [2]. True triaxial compression tests differ from conventional triaxial tests because the magnitudes of both the intermediate and the minor principal stresses are independently controlled. In a standard triaxial test the mean effective stress p' typically increases. In a drained true triaxial test it is common to keep both the mean effective stress and the intermediate principal stress ratio (b) constant where

$$
b=\frac{\sigma_{2}^{\prime}-\sigma_{3}^{\prime}}{\sigma_{1}^{\prime}-\sigma_{3}^{\prime}}
$$

In this way, the effect of $\mathrm{b}$ on the stress-strain behaviour can be isolated. In true triaxial compression $\mathrm{b}=0$ and during shearing $\sigma_{1}^{\prime}$ increases while $\sigma_{2}^{\prime}$ and $\sigma_{3}^{\prime}$ both decrease equally at a rate that is half the rate of increase of $\sigma_{1}^{\prime}$ in order to keep p' constant.

It is clear from previous DEM studies $[2,23]$ and from previous experimental research [24 - 27], that as $b$ increases from the triaxial condition $(b=0.0)$ towards the plane strain condition $\left(b_{P S} \cong 0.5\right)$ the angle of shearing resistance $\phi_{\text {peak }}$ also increases. However $a$ s $b$ continues to increase beyond the plane strain condition to achieve conditions similar to those experienced during triaxial extension $(b=1.0)$ there is no consensus on the effects of $\mathrm{b}$ on $\phi_{\text {peak. }}$. DEM research $[2,23]$ shows that $\phi_{\text {peak }}$ decreases from $b_{P S}$ to $b=1.0$ and also state that the resistance in triaxial compression $(b=0)$ is lower than in triaxial extension 
$(b=1)$. Experimental research carried out by Haruyama [25] and Sutherland and Mesdari [27] seems to agree with these numerical results. However, tests by Green [26] and Shapiro and Yamamuro [28] indicate that there is no effect of $b$ on $\phi_{\text {peak }}$ when $b \geq b_{P S}$. The reason for the discrepency in observations is difficult to explain. Perhaps the differences arise because different authors tested different sands and it is difficult to guarantee that the initial state of every sample is the same. Furthermore, the true triaxial apparatusses used by these researchers use different combinations of boundary types including rigid platens, flexible membranes or combinations of both. The use of DEM and periodic boundaries in particular overcomes these difficulties.

In the current study a series of simulations, each with a constant p' of $200 \mathrm{kPa}$ and using different values of $\mu$ and $b$ were performed. Typical responses of the principal stresses for each simulation are presented in Figure 6. For each of these tests the initial specimen configuration was identical, with a void ratio of 0.521 and an initial isotropic fabric. Figure 6(a) indicates that, for each test, as the specimen is sheared $\sigma_{1}^{\prime}$ increases to a peak value $\left(\sigma_{1}^{\prime}{ }^{\text {peak }}\right)$ and exhibits a slight post-peak strain softening, and there is a consistent trend for $\sigma_{1}^{\prime}{ }^{\text {peak }}$ to decrease with $\mathrm{b}$. Similarly referring to Figure $6(\mathrm{c}), \sigma_{3}^{\prime}$ decreases to a minimum value with magnitude of $\sigma_{3}^{\prime \min }$ and there is a subsequent post-peak increase in $\sigma_{3}^{\prime}$, and the magnitude of $\sigma_{3}^{\prime}{ }^{\text {min }}$ decreases with increasing $b$. The trend in the variation of $\sigma_{2}^{\prime}$ is more complex. Referring to Figure $6(\mathrm{~b})$ for $0 \leq \mathrm{b}<0.5$, upon shearing $\sigma_{2}^{\prime}$ reduces and the magnitude of the minimum $\sigma_{2}^{\prime}$ value gradually decreases (i.e. $\sigma_{2}^{\prime}{ }^{\min }$ increases) as $\mathrm{b}$ increases. Then for $\mathrm{b}=0.5$ no change in $\sigma_{2}^{\prime}$ is observed during shearing. There is then a transition to a response where $\sigma_{2}^{\prime}$ increases during shearing, and for $0.5<b<1.0$, the magnitude of $\sigma_{2}^{\prime \max }$ increases with increasing $b$. When $b=1, \sigma_{1}^{\prime}=\sigma_{2}^{\prime}$ and their magnitudes increase at a rate equal to half the rate of decrease of $\sigma_{3}^{\prime}$. These conditions (b $=1)$ are similar to those experienced in conventional triaxial extension tests but differ in the sense that the orientation of the major principal stress is aligned with the vertical direction and the sample is compressed vertically. 
The data presented in Figure 6 are unified in Figure 7(a) where the variation in the deviator stress $t$ with deviatoric strain is presented. It is clear that the maximum peak $t$ value is mobilized in the simulation with $\mathrm{b}=0.4$ while the peak mobilized $\mathrm{t}$ value is a minimum for $b=1.0$. This is in agreement with all DEM and experimental studies available. The difference in the initial shear stiffness seems to be independent of $b$, at least for the strain levels observed here. Figure 7(b) shows that the influence of the $b$ value on the volumetric response is negligible, and as expected all the specimens experienced an overall dilation during shear. Figure 8 demonstrates that the normal (or direct) strain response along each of the principal stress orientations differs. Figure 8(a) shows that $\varepsilon_{1}$ is always compressive and that the rate of compression decreases as $b$ increases. Similarly $\varepsilon_{3}$ in Figure 8(c) is always extensive and the rate of expansion increases with increasing $b$. Note however that as b increases the resultant increment in the rate of expansion becomes less significant. As shown in Figure $8(\mathrm{~b})$ in the $\sigma_{2}^{\prime}$ direction the normal strain response is highly dependent on the $b$-value. When $b=0.4$ the specimen does not extent or contract significantly, i.e. there is a response very close to plane strain (PS). If $b>b_{\mathrm{PS}}$ the $\varepsilon_{2}$ response is compressive. On the contrary, if $\mathrm{b}<\mathrm{b}_{\mathrm{PS}}$ then $\varepsilon_{2}$ shows an extensional behaviour. The overall strain response illustrated in Figure 8 is consistent with the stress behaviour discussed with reference to Figure 6. Note that for this set of simulations $b_{\mathrm{PS}}$ (nor contraction or dilation) does not occur exactly when $\sigma_{2}^{\prime}$ is kept constant $(b=0.5)$. The current data indicates that stress conditions in plane strain are highly dependent on the value of friction, so it is expected that $b_{\mathrm{PS}}=0.5$ for a particular value of $\mu$ only.

Experimental evidence regarding the strain response of soil samples in true triaxial tests is as controversial as the variations of $\phi^{\text {peak }}$. Most researchers [26 - 27, $\left.29-31\right]$ agree that axial strains to failure $\left(\varepsilon_{1}{ }^{f}\right)$ decrease when b increases. However, Reades and Green [32] describe an initial increase on $\varepsilon_{1}{ }^{\mathrm{f}}$ when $0<\mathrm{b}<0.2$ and then a decrease when $\mathrm{b}>0.2$. Furthermore, Lade and Duncan [29] also found that this variation is dependent on the 
initial state of the sample. Hence, comparison of experimental results and the DEM results presented here is inconclusive. Note however, that according to Figures 7 and 6(a), $\varepsilon_{1}{ }^{\mathrm{f}}$ appears to increase slightly as b increases. In terms of $\varepsilon_{\mathrm{v}}$ Reades and Green [32]

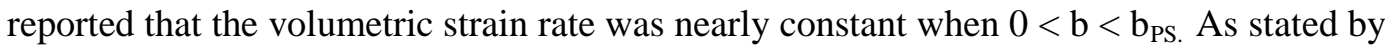
Lade et al. [19] the volumetric responses of physical experiments are affected by the boundary conditions but the results in Figure 7(b) seem to support the idea of $\varepsilon_{\mathrm{v}}$ being independent of $\mathrm{b}$ and agree with results presented by Thornton and Zhang [3] from DEM simulations.

Figure 9 gives the evolution of $\mathrm{G}_{\text {oct }}$ for the same set of simulations. Figure 9 (a) shows that the effect of $b$ on $G_{\text {oct }}$ is negligible and this is confirmed in Figure $9(b)$ where the data are presented following the format of Figure 2(b). However while the data in Figure 2(b) clearly showed the influence of $\mu$ on $\mathrm{G}_{\text {oct }}$, the data presented in Figure 9(b) show that the effect of $b$ on $\mathrm{G}_{\mathrm{oct}}$ is insignificant.

The influence of $\mu$ on the relationship between the peak angle of shearing resistance $\phi_{\text {peak }}$ $\left[=\sin ^{-1}\left(\left(\sigma_{1}^{\prime}-\sigma_{3}^{\prime}\right) /\left(\sigma_{1}^{\prime}+\sigma_{3}^{\prime}\right)\right)\right]$ and $\mathrm{b}$ was explored and, as illustrated in Figure $10(\mathrm{a}), \phi_{\text {peak }}$ clearly increases with $\mu$ for all the b-values considered. There is a clear consistent trend in the pattern of response, as confirmed in Figure 10(b) where the responses for each $\mu$ value are seen to be indistinguishable when normalized by $\phi_{\text {peak }}$ for $b=0.0$. This result is not surprising if we accept that $\mu$ affects the inherent stability of the strong force network, while the level of support provided by the weak force network is only slightly affected by friction as demonstrated in Figure 4(b).

Prior research has considered the sensitivity of the material strength to $\mu$ for triaxial conditions, and Figure 11 compares the results of this study with previously published data in terms of $\sin \left(\phi_{\text {peak }}\right)$. The trends observed here for the constant $b$ tests agree with the observations by Thornton [2] for triaxial compression response. The range of variation of attainable $\sin \left(\phi_{\text {peak }}\right)$ values increases when the more general true triaxial stress conditions 
are considered and it seems that a limiting envelop can be found when $b=0.5$ (i.e. when $\left.\mathrm{b} \approx \mathrm{b}_{\mathrm{PS}}\right)$. Also note that true triaxial simulations $(\mathrm{b}=0)$ give the same $\sin \left(\phi_{\text {peak }}\right)$. When interpreted in relation to the t-p' space, during true triaxial compression the stress path is vertical (as p' is kept constant), while in conventional triaxial compression the stress path is inclined. However both stress paths approach the same strength envelope in the generalised stress space and the orientation of the stress path in the generalised stress plane is determined by $b$ (a function of Lode's angle, equal for both tests).

Lade and Duncan [33] proposed that a parameter $\eta$ determines the position of the strength envelope in the generalised stress space and $\eta$ is given by:

$$
\eta=\left(\frac{I_{1}^{3}}{I_{3}}-27\right) \cdot\left(\frac{I_{1}}{p_{a}}\right)^{m}
$$

where $I_{1}$ and $I_{3}$ are the first and third invariants of the stress tensor, $p_{a}$ is the atmospheric pressure in the same units of the stress and $m$ is the slope of the best-fit line when plotting $\left(I_{1}^{3} / I_{3}-27\right)$ versus $\left(\mathrm{p}_{\mathrm{a}} / \mathrm{I}_{1}\right)$. Figure 12 illustrates the results in the generalised stress plane and the responses agree with the Lade and Duncan failure criterion. Previous DEM simulations by Ng [1] Thornton [2] and Thornton and Zhang [3] also showed agreement with the Lade and Duncan failure criterion. Here $\eta$ is seen to be a function of $\mu$ (Figure 13(a)) with $\eta=14.789 \mu^{0.3754}$ It can therefore be implied that the value of $\eta$ determined from physical true triaxial tests on a sand is an index of the inherent stability of the force chains in that sand. It is likely that correlations could be found between $\eta$ and the sand mineralogy (which will determine the friction) or, more likely, between $\eta$ and the particle geometry (more angular, less convex particles will form more stable force chains).

When Thornton [2] analysed the results of constant b-value tests he proposed that a peak fabric envelope could be represented using an "inverted" Lade \& Duncan envelope described by: 


$$
\eta^{*}=\frac{3 \phi_{1}^{3}}{2 \phi_{1} \phi_{2}-3 \phi_{3}}
$$

where $\Phi_{1}, \Phi_{2}$ and $\Phi_{3}$, are the principal values of the fabric tensor. The peak fabric envelopes for the simulations in the current study are also included in Figure 12. While the range of $\eta^{*}$ values is smaller than the range of $\eta$ values, there is a relationship between $\eta^{*}$ and $\mu$ and the form of this relationship is similar to the relationship between $\eta$ and $\mu$, in this case $\eta^{*}=5.488 \mu^{0.0034}$ as illustrated in Figure 13(b). The added stability provided to the contacts by increasing $\mu$ therefore has a small, but consistent influence on the material fabric.

Figure 14(a) shows the evolution of the total number of contacts (i.e. $\mathrm{N}_{c}$, rather than $\mathrm{Z}$ ) belonging to the force network for true triaxial tests with various $b$-values for $\mu=0.14$ and $\mu=0.7$. Figure 14 (b) does the same but considering the strong force network only. An increase of $\mu$ results in a reduction in the number of contacts. Note that $\mathrm{N}_{\mathrm{c}}{ }^{\mathrm{s}}$ seems to be increasing after reaching $\mathrm{N}_{\mathrm{c} \text { min }}^{\mathrm{s}}$ while $\mathrm{N}_{\mathrm{c}}$ appears to be constant. However, the number of contacts seems to be unaffected by b, i.e. it is solely a function of $\mu$. As noted above $G_{\text {oct }}$ varied with $\mu$ but not with $b$; Figure 14 implies that $\mathrm{G}_{\mathrm{oct}}$ is then dependant on the number of contacts in the force network, explaining the lack of sensitivity of $b$ to $G_{\text {oct }}$.

To measure the variation in fabric for this three dimensional stress state a generalised octahedral fabric can be defined as:

$$
\phi_{\text {oct }}=\frac{1}{\sqrt{2}}\left[\left(\phi_{1}-\phi_{2}\right)^{2}+\left(\phi_{2}-\phi_{3}\right)^{2}+\left(\phi_{1}-\phi_{3}\right)^{2}\right]^{0.5}
$$

where $\Phi_{1}, \Phi_{2}$ and $\Phi_{3}$ are the principal values of $\Phi_{\mathrm{ij} \cdot}$. This expression reduces to $\Phi_{\mathrm{d}}=\Phi_{1}$ $\Phi_{3}$ for triaxial (axi-symmetric) conditions as proposed by Thornton [2]. Figure 15 shows the evolution of $\Phi_{\text {oct }}$ for a set of true triaxial tests with $\mu=0.14$ and $\mu=0.7$. A qualitative agreement to the macro-scale stress-deformation behaviour is observed. The smallest 
amount of anisotropy is induced when $b=0.0$ (providing the lowest shear strength) and the greatest amount of anisotropy is induced when $b=0.4$, which is reasonably close to

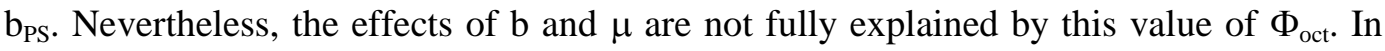
fact, it is clear that when analysed in this manner, the effects of $b$ appear to be insignificant in comparison to those of friction. Consequently it is useful, as before, to isolate the response of the strong and weak force network. The evolution of $\Phi_{i}{ }^{\mathrm{s}}$ is provided in Figure 16 for two sets of constant $b$ tests with two different values of $\mu$. Figures 16(a), 16(c) and 16(e) illustrate $\Phi_{1}{ }^{\mathrm{s}}, \Phi_{2}{ }^{\mathrm{s}}$ and $\Phi_{3}{ }^{\mathrm{s}}$ respectively when $\mu=0.14$. Similarly, Figures $16(\mathrm{~b}), 16(\mathrm{~d})$ and $16(\mathrm{f})$ do the same for $\mu=0.7$. Independently of $\mu$ there is a clear decrease in $\Phi_{1}{ }^{\mathrm{s}(\max )}$ and $\Phi_{3}{ }^{\mathrm{s}(\min )}$ as b increases. For $\Phi_{2}{ }^{\mathrm{s}}$ there are two types of observed behaviour. When $0<\mathrm{b}<\mathrm{b}_{\mathrm{PS}}$ the value of $\Phi_{2}{ }^{\mathrm{s}}$ reduces and for $\mathrm{b}>\mathrm{b}_{\mathrm{PS}}$ its value increases. Hence, there is a good correlation between the strong fabric changes and the changes in principal stresses and strains in Figures 6 and 8. Also note however, that the bvalue required to achieve plane strain conditions (in terms of fabric) is also dependent on the value of $\mu$. In agreement with Figure 1(a) and 4(a), high values of $\mu$ provide a "stiffer" evolution of $\Phi_{\mathrm{i}}^{\mathrm{s}}$ than low friction values. Note that for both low and high values of $\mu$ the magnitudes of the $\Phi_{i}{ }^{\mathrm{s}}$ values are similar, but as discussed before fewer contacts are required to achieve this anisotropy when $\mu$ is high. This strongly supports the fact that friction provides a significant stability to the strong force chains. Interestingly, when $\mu=$ 0.14 (Figure 16(e)) the value of $\Phi_{3}{ }^{\mathrm{s}}$ min occurs when $\mathrm{b}>0.6$, while for $\mu=0.7$ (Figure 16(f)) the limit $\Phi_{3 \text { min }}^{\mathrm{s}}$ is reached when $\mathrm{b}>0.4$. As was the case in triaxial compression, the weak fabric remains almost isotropic during shearing and the orientation of the principal fabric $\beta^{\Phi}{ }_{s}$ coincides with that of the major principal stress $\beta^{\sigma}$, but $\beta^{\Phi}{ }_{w}$ cannot be calculated in a meaningful way as it is approximately isotropic.

It is important to illustrate clearly the role of the contacts orthogonal to the strong force network in providing support. It can be appreciated by reference to Figures 17 and 18 which show polar histograms of the projection of contact orientations in the horizontal 
plane (parallel to the orientations of $\sigma_{2}^{\prime}$ and $\sigma_{3}^{\prime}$ ) for $\mu=0.14$ and $\mu=0.70$ respectively. While the radial length of each angular bin indicates the number of contacts oriented in that direction, the shading indicates the average magnitude of the contact forces for that bin normalised by the average magnitude of the contact forces in the specimen. It is clear from these figures that forces with a relatively large magnitude develop in the lateral plane and the shape of this distribution varies with $b$. It seems that for low $b$ values there is an isotropic distribution of forces providing support to the strong force network. As b increases there are slightly more and stronger forces in the y $\left(\sigma_{2}^{\prime}\right)$ direction, transmitting larger forces that are themselves more stable, giving more support to the strong force chains, while at the same time the support offered in the $\mathrm{x}\left(\sigma_{3}^{\prime}\right)$ direction still exists and only slightly reduced for $b<0.5$. When $b$ approaches 1.0 , the increased support offered in the $\mathrm{y}$-direction is counter acted by the reduced support offered in the $\mathrm{x}$-direction, resulting in lower $\phi_{\text {peak }}$ values.

\section{Discussion and conclusions}

This paper has made a contribution to advance fundamental understanding of granular material response by considering both the effects of friction and stress state on the material response. Using DEM simulations of triaxial compression and true triaxial compression tests we have presented alternative ways to interpret the evolution of fabric in order to link the evolution of micro-mechanical parameters to the macro-scale behaviour of granular materials. We have considered the effect of the inter-particle friction $(\mu)$ and the intermediate stress ratio (b) on the granular material's response.

The main findings from the triaxial compression tests were:

1. Considering the macro-scale load-deformation response in the triaxial test simulations, in line with previous studies, as $\mu$ increases there are corresponding increases in the peak angle of shearing resistance $\left(\phi^{\text {peak }}\right)$, the 
amount of dilation (i.e. negative volumetric strain, $\varepsilon_{\mathrm{v}}$ ) and the shear stiffness $\left(\mathrm{G}_{\mathrm{oct}}\right)$. There is a non-linear relationship between $\mu$ and each of these response characteristics, reflecting the complexity of the material response. The mechanism of force chain failure most likely explains the differences in stiffness and strain at which the peak stress is mobilized.

2. Analysis of the particle-scale data within the DEM simulations revealed that as $\mu$ increases, the critical coordination number decreased and there was an increase in the magnitude of the deviatoric fabric induced during shearing. The critical coordination number that specimens attain after a small amount of straining does not indicate a steady state response. In fact, examination of the fabric tensor data revealed that the constant $\mathrm{Z}$ values indicate that the addition of contact to the strong force network that is aligned with the major principal stress, is balanced by the removal of contacts from the orthogonal weak force network. Furthermore, analysis of the evolution of $Z \Phi_{d}$ demonstrated that the number of contacts participating in the strong force chains is relatively insensitive to $\mu$. This data also indicate that there is not a one-one functional relationship between $\mathrm{Z}$ and e, rather $\mathrm{Z}$ depends on $\Phi_{\mathrm{d}}$ as well as $\varepsilon$.

3. Decomposition of the fabric tensor in its strong and weak components demonstrated strong force chains can collapse in a gradual manner at low friction values, while under a high friction regime the collapse could occur almost suddenly when the tangential force of most of the strong contacts attains a limiting value. While the strong fabric is orientated in the direction of the principal stress, the weak fabric remains largely isotropic and it was only slightly affected by the value of $\mu$.

The main findings from the true triaxial compression (constant p', constant $b$ ) test simulations can be summarized as follows: 
1. Both $b$ and $\mu$ affect the macro-scale response in different ways; the value of $\mu$ determines the inherent stability of the force chains while the value of $b$ determines the amount of external, lateral support.

2. While the peak stress mobilized is a function of both $\mathrm{b}$ and $\mu$, the stiffness, $\mathrm{G}_{\mathrm{oct}}$, is independent of $\mathrm{b}$.

3. There appears to be a unique relationship between $\mathrm{b}$ and $\phi_{\text {peak }} / \phi_{\text {peak } b=0}$.

4. The $\eta$ parameter used to define the shape of the Lade and Duncan (1975) failure criterion in principal stress space is a function of $\mu$. As $\mu$ determines the stability of the strong force chains, the value of $\eta$ obtained in physical experiments on a given sand is a measure of the stability of the force chains that form in that sand.

5. There is a functional correlation (with a low sensitivity) between the $\eta^{*}$ parameter that defines the shape of the fabric response envelops and $\mu$, this is evidence that friction also affects the geometrical fabric and does not simply add to the stability of force networks with the same topologies.

6. There is a clear link between the octahedral fabric measure that considers the influence of the intermediate principal fabric and the macro-scale behaviour.

\section{Acknowledgements}

Financial support for the study was provided by grant EP/D50631X/1 from the Engineering and Physical Sciences Research Council. The financial support provided by the Department of Civil and Environmental Engineering, Imperial College London is also kindly acknowledged.

\section{References}

1. Ng, T.-T.: Behaviour of gravity deposited granular material under different stress paths. Canadian Geotechnical Journal, 42(6), 1644-1655 (2005)

2. Thornton, C.: Numerical simulations of deviatoric shear deformation of granular media. Géotechnique, 50(1), 43-53 (2000)

3. Thornton, C., and Zhang, L.: On the evolution of stress and microstructure during general 3D deviatoric straining of granular media. Géotechnique, 60(5), 333-341 (2010)

4. Yimsiri, S., and Soga, K.: DEM analysis of soil fabric effects on behaviour of sand. Géotechnique, 60(6), 483-495 (2010) 
5. Barreto, D.: Numerical and experimental investigation into the behaviour of granular materials under generalised stress states. Ph.D. thesis, Imperial College of Science, Technology and Medicine, University of London (2009)

6. Lin, X., and Ng, T.-T.: A three-dimensional discrete element model using arrays of ellipsoids. Géotechnique, 47(2), 319-329 (1997)

7. Cundall, P.A. and Strack, O.D.L.: The distinct element method as a tool for research in granular media, Part II. Technical report. University of Minnesota. (1979)

8. O'Sullivan, C.: The application of discrete element modelling to finite deformation problems in geomechanics. PhD thesis. University of California, Berkeley. (2002)

9. Cui, L.: Developing a virtual test environment for granular materials using discrete element modelling. PhD thesis. University College Dublin. (2006)

10. O'Sullivan, C.: Particulate Discrete Element Modelling: A Geomechanics perspective. Spon Press (2011)

11. Satake, M.: Fabric tensor in granular materials. In Proceedings IUTAM Conference on Deformation and Failure of Granular Materials, Delft, pp. 63-67 (1982)

12. Muthuswamy, M., and Tordesillas, A.: How do interparticle friction, packing density and degree of polydispersity affect force propagation in particulate assemblies? Journal of Statistical Mechanics: Theory and Experiment, 2006(9), P09003, (2006)

13. Radjai, F., Wolf, D.E., Jean, M., and Moreau, J.-J.: Bimodal Character of Stress Transmission in Granular Packings. Physical Review Letters, 80(1), 61-64, (1998)

14. Tordesillas, A., Shi, J., and Tshaikiwski, T.: Stress-dilatancy and force chain evolution. International Journal for Numerical and Analytical Methods in Geomechanics, 35(2), 264-292, (2011)

15. Tordesillas, A., and Muthuswamy, M.: A thermomechanical approach to multiscale continuum modelling of dense granular materials. Acta Geotechnica, 3(3), 225-240, (2008)

16. Oda, M.: Co-ordination number and its relation to shear strength of granular materials. Soils and Foundations, 17(2), 29-42 (1977)

17. Santamarina, J.C., and Cascante, G.: Stress anisotropy and wave propagation: a micromechanical view. Canadian Geotechnical Journal, 33(5), 770-782 (1996)

18. Hasan, A., and Alshibli, K.: Experimental assessment of 3D particle-to-particle interaction within sheared sand using synchrotron microtomography. Géotechnique 60(5), 369-379 (2010)

19. Thornton, C., and Anthony, S.J.: Quasi-Static Deformation of Particulate Media.

Philosophical Transactions Royal Society of London A: Mathematical, Physical and Engineering Sciences, 356(1747), 2763-2782 (1988)

20. Skinner, A.E.: A note on the influence of interparticle friction on the shearing strength of a random assembly of spherical particles. Géotechnique, 19(1), 150-157 (1969)

21. Cavarreta, I.: The influence of particle characteristics on the engineering behaviour of granular materials. Ph.D. thesis, Imperial College of Science, Technology and Medicine, University of London (2009) 
22. Powrie, W., Ni, Q., Harkness, R.M., and Zhang, X.: Numerical modelling of plane strain tests on sands using a particulate approach. Géotechnique, 55(4), 297-306 (2005)

23. Ng, T.-T.: Macro- and micro-behaviours of granular materials under different sample preparation methods and stress paths. International Journal of Solids and Structures, 41(21), 58715884 (2004)

24. Lade, P.V., Nam, J., and Hong, W.P.: Shear banding and cross-anisotropic behaviour observed in laboratory sand tests with stress rotation. Canadian Geotechnical Journal, 45(1), 74-84 (2008)

25. Haruyama, M.: Anisotropic deformation-strength characteristics of an assembly of spherical particles under three dimensional stresses. Soils and Foundations, 21(4), 41-55 (1981)

26. Green, G.E.: Strength and deformation of sand measured in an independent stress control cell. In Proceedings of the Roscoe Memorial Symposium, Cambridge, England, Foulis and Co., Ltd, pp. 285-323 (1971)

27. Sutherland, H.B., and Mesdari, M.S.: The influence of the intermediate principal stress on the strength of sands. In Proceedings of the $7^{\text {th }}$ International Conference on Soil Mechanics and Foundation Engineering, Mexico City, Mexico, Vol. 1, pp. 391-399 (1969)

28. Shapiro, S., and Yamamuro, J.A.: Effect of silt on three-dimensional stress-strain behaviour of loose sand. Journal of Geotechnical and Geonvironmental Engineering, ASCE. 129(1), 1-11 (2003)

29. Lade, P.V., and Duncan, J.M.: Cubical triaxial tests on cohesionless soil. Journal of the Soil Mechanics and Foundations Division, ASCE. 99(10), 793-812 (1973)

30. Symes, M.J.P.R.: Rotation of principal stresses in sand. Ph.D. thesis, College of Science, Technology and Medicine, University of London (1983)

31. Wang, Q., and Lade, P.V.: Shear banding in true triaxial tests and its effect on failure in sand. Journal of Geotechnical and Geonvironmental Engineering, ASCE. 127(8), 754-761. (2001)

32. Reades, D.W., and Green, G.E.: Independent stress control and triaxial extension tests on sand. Géotechnique, 26(4), 551-576 (1976)

33. Lade, P.V., and Duncan, J.M.: Elastoplastic stress-strain theory for cohesiveless soil. Proceedings American Society of Civil Engineers, Journal of Geotechnical Engineering, 101(10), 1037-1053 (1975)

\section{Figure captions}

Fig. 1 Influence of friction on (a) stress-strain response, (b) overrall volumetric behaviour and (c) volumetric behaviour at small strains during triaxial compression.

Fig. 2 Influence of friction on (b) the evolution of the octahedral stiffness and (b) its value at various strain levels in triaxial compression 
Fig. 3 Influence of friction on the evolution of (a) the coordination number, and (b) the deviatoric fabric, (c) the product of $Z$ and $\Phi_{d}$ and (d) evolution of $Z$,e and $\Phi_{d}$ in triaxial compression

Fig. 4 Influence of friction on the (a) strong and (b) weak fabric in triaxial compression while considering the major and minor principal values

Fig. 5 Contact normal forces on the XZ-plane for peak states of triaxial compression simulations with (a) $\mu=0.01$ and (b) $\mu=0.50$

Fig. 6 Variation of the (a) major, (b) intermediate and (c) minor principal stresses for true triaxial tests with $\mu=0.22$ and different $b$ values

Fig. 7 Influence of the b-value on (a) stress-strain and (b) volumetric response in true triaxial compression with $\mu=0.22$

Fig. 8 Effect of the b-value on the (a) major, (b) intermediate and (c) minor principal strains during true triaxial compression with $\mu=0.22$

Fig. 9 Influence of the b-value on (b) the evolution of the octahedral stiffness and (b) its value at various strain levels in true triaxial compression with $\mu=0.22$

Fig. 10 Influence of inter-particle friction on the (a) peak angle of shearing resistance and (b) the normalised peak shear resistance in true triaxial compression

Fig. 11 Influence of inter-particle friction and the intermediate stress ratio on the angle of shearing resistance in relation to previous studies

Fig. 12 Influence of inter-particle friction on the peak stress and fabric states for true triaxial compression in the generalised stress plane

Fig. 13 Effect of inter-particle friction on (a) the Lade \& Duncan (1975) $\eta$ parameter and (b) Thornton (2000) $\eta^{*}$ parameter

Fig. 14 Effect of $\mu$ and $b$ on the number of contacts in the (a) force network and (b) the strong force network

Fig. 15 Effect of the intermediate stress ratio on the octahedral fabric for two values of friction

Fig. 16 Effect of the inter-particle friction on the strong force chains in true triaxial compression for two values of friction

Fig. 17 Effect of $b$ on the orthogonal support to the strong force chains for $\mu=0.14$

Fig. 18 Effect of $b$ on the orthogonal support to the strong force chains for $\mu=0.70$ 

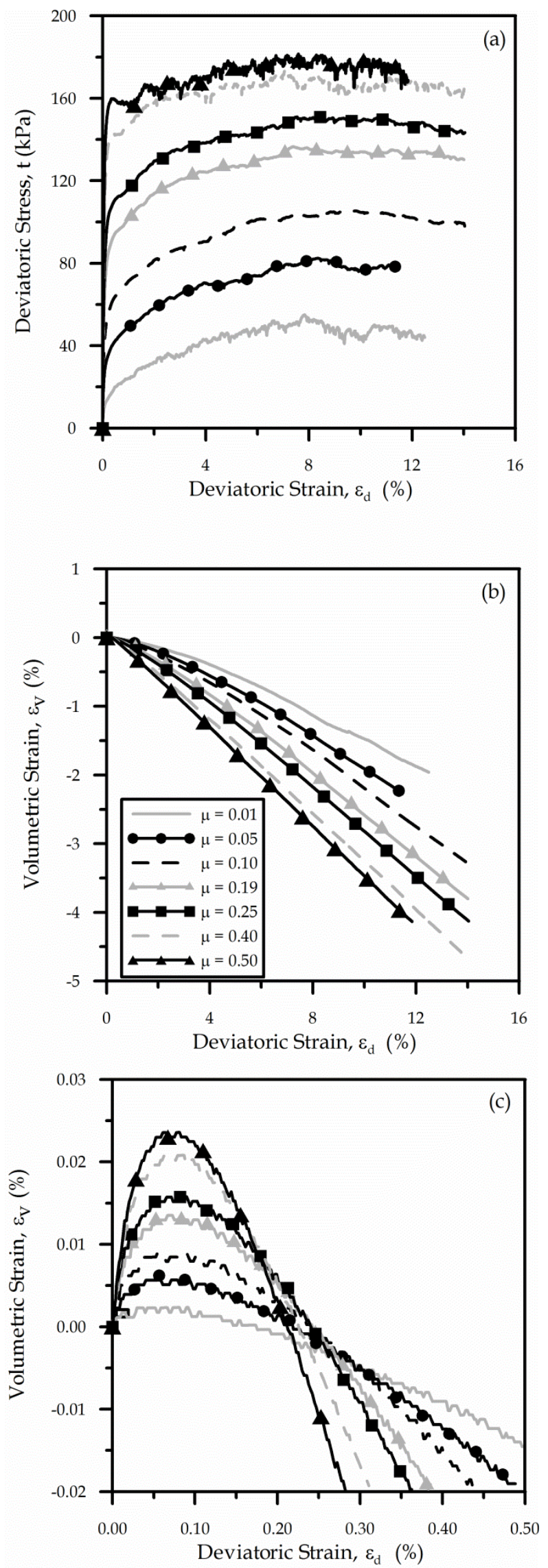

Figure 1 

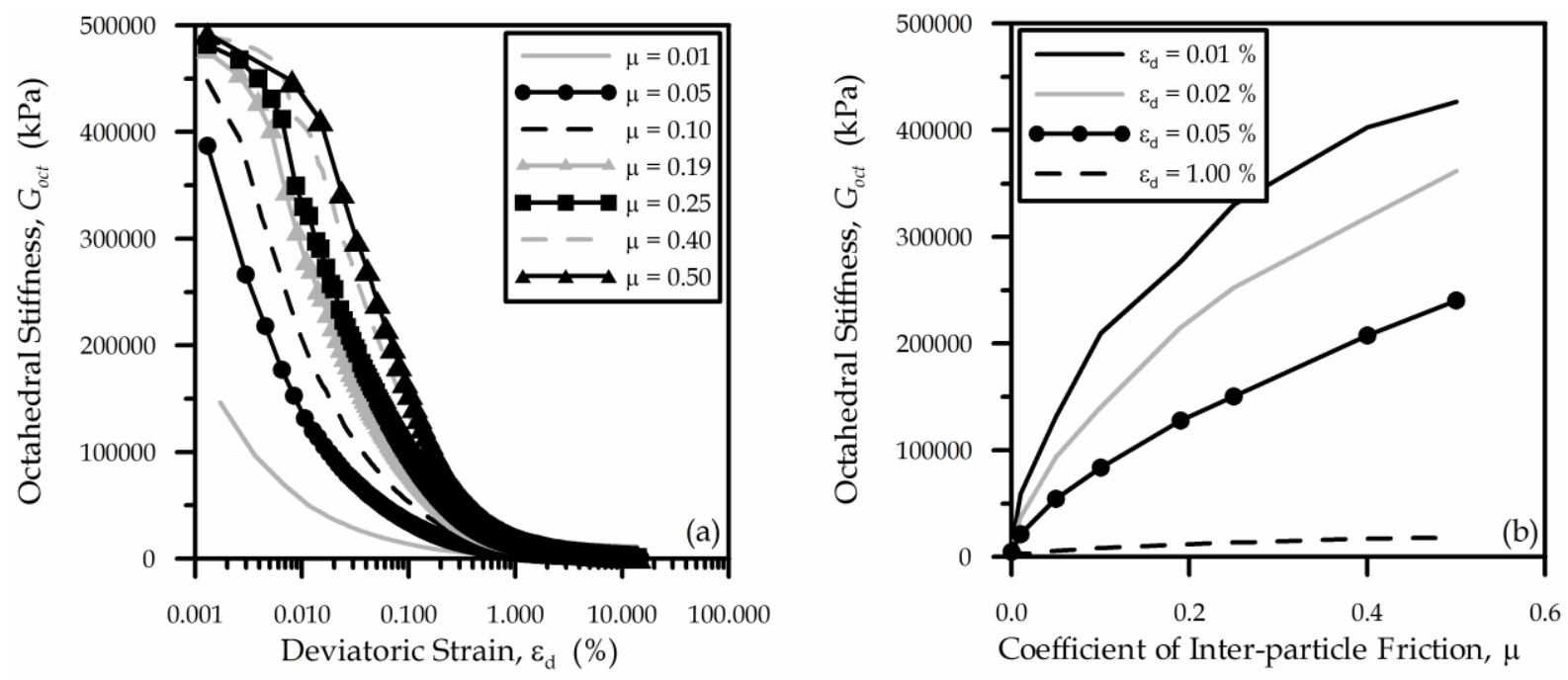

Figure 2 

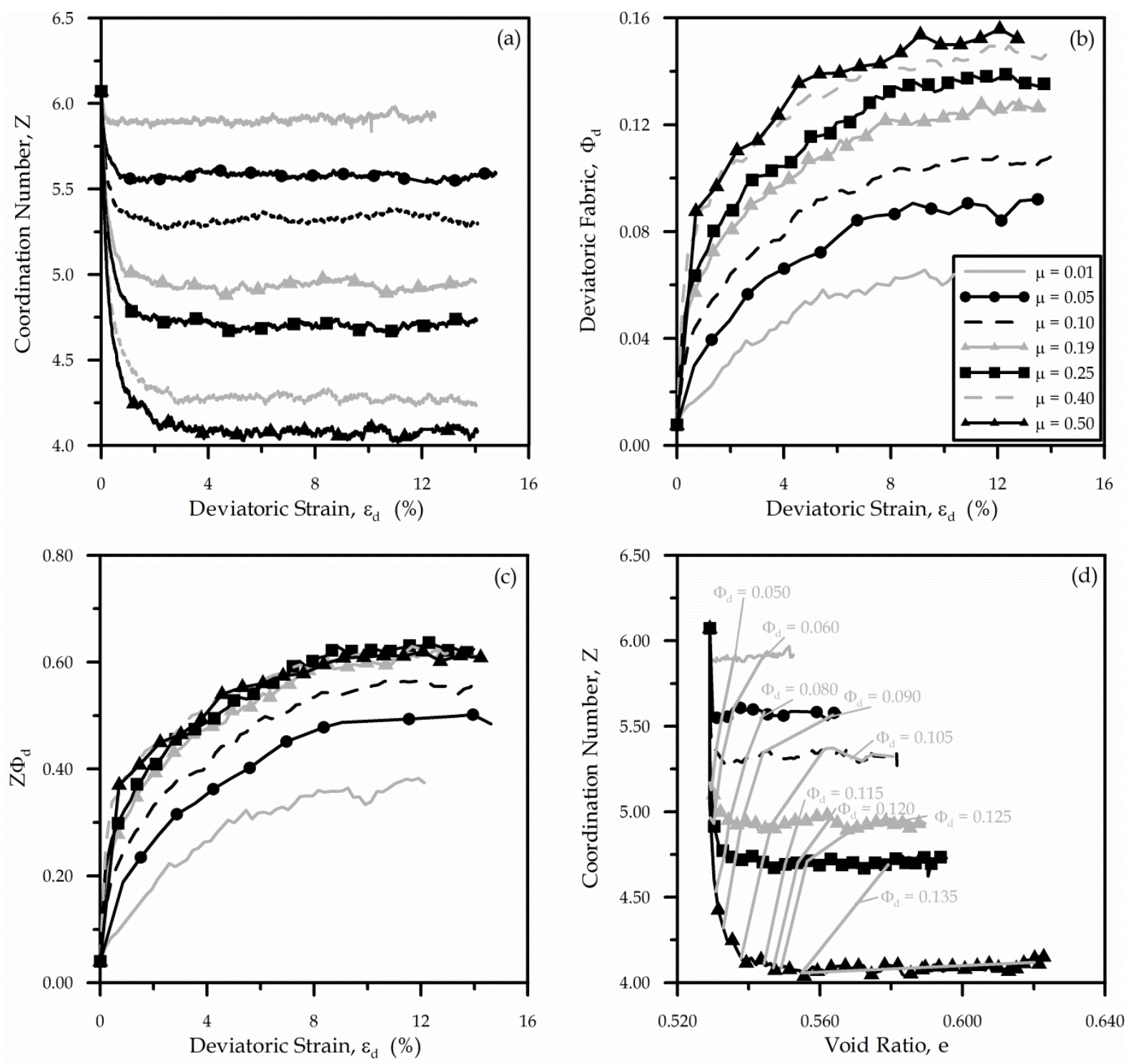

Figure 3 

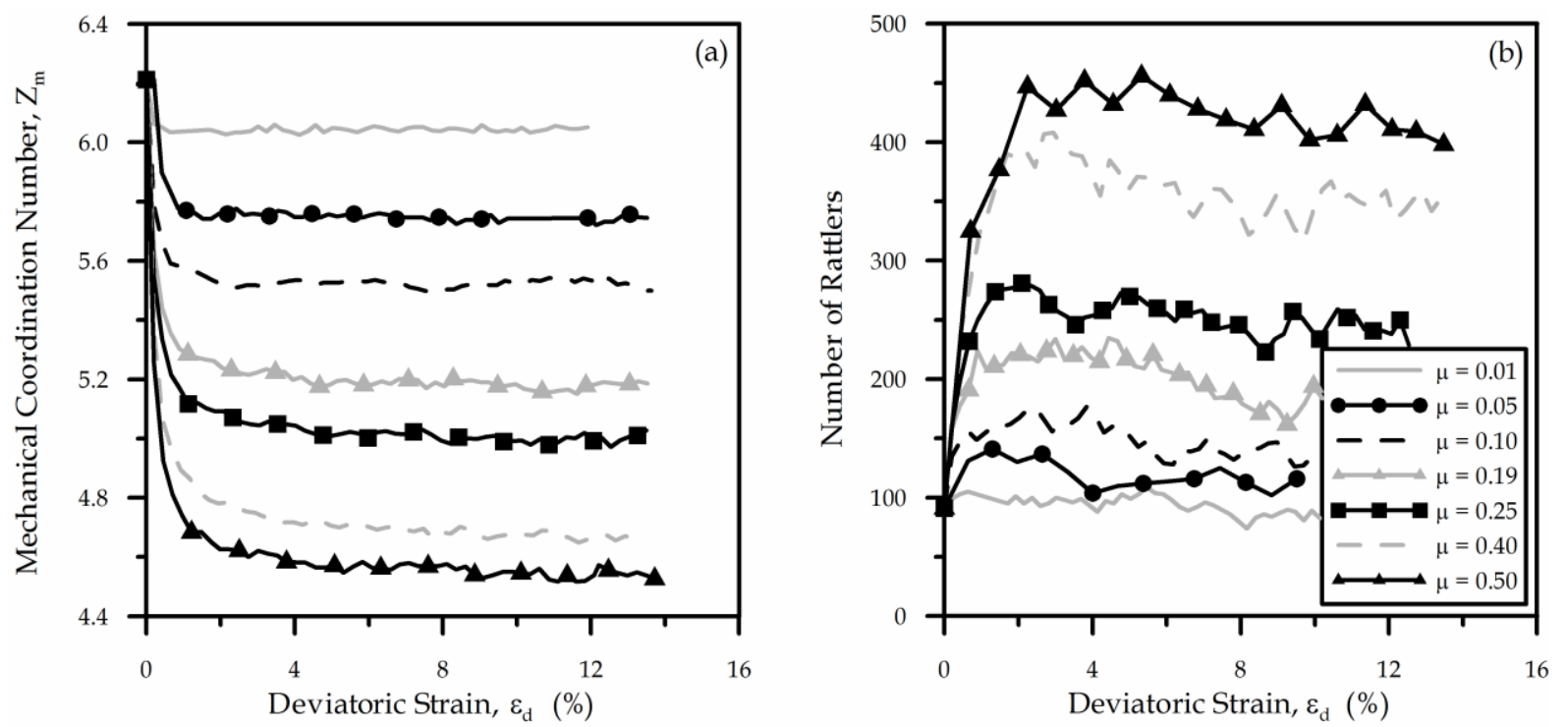

Figure X 

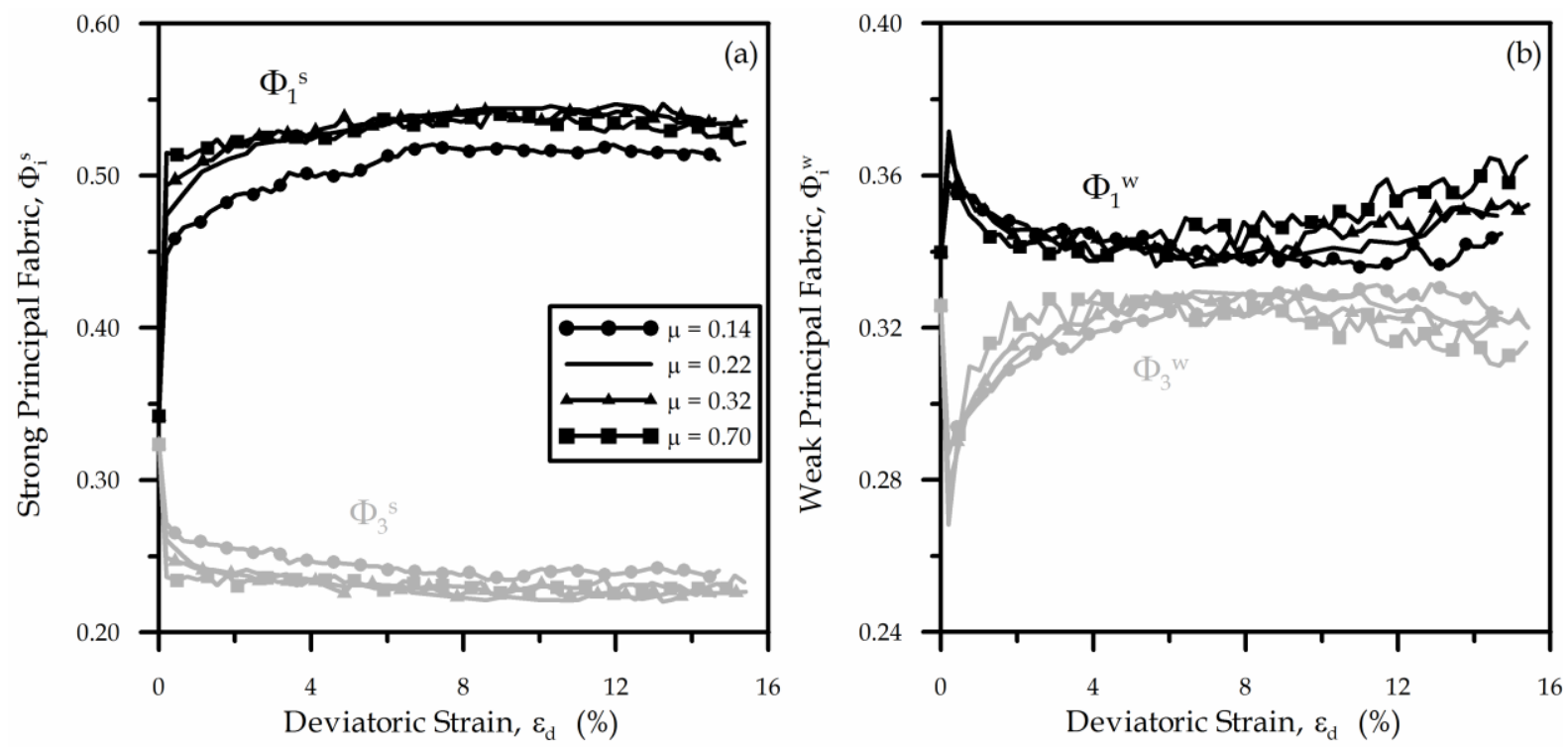

Figure 4 

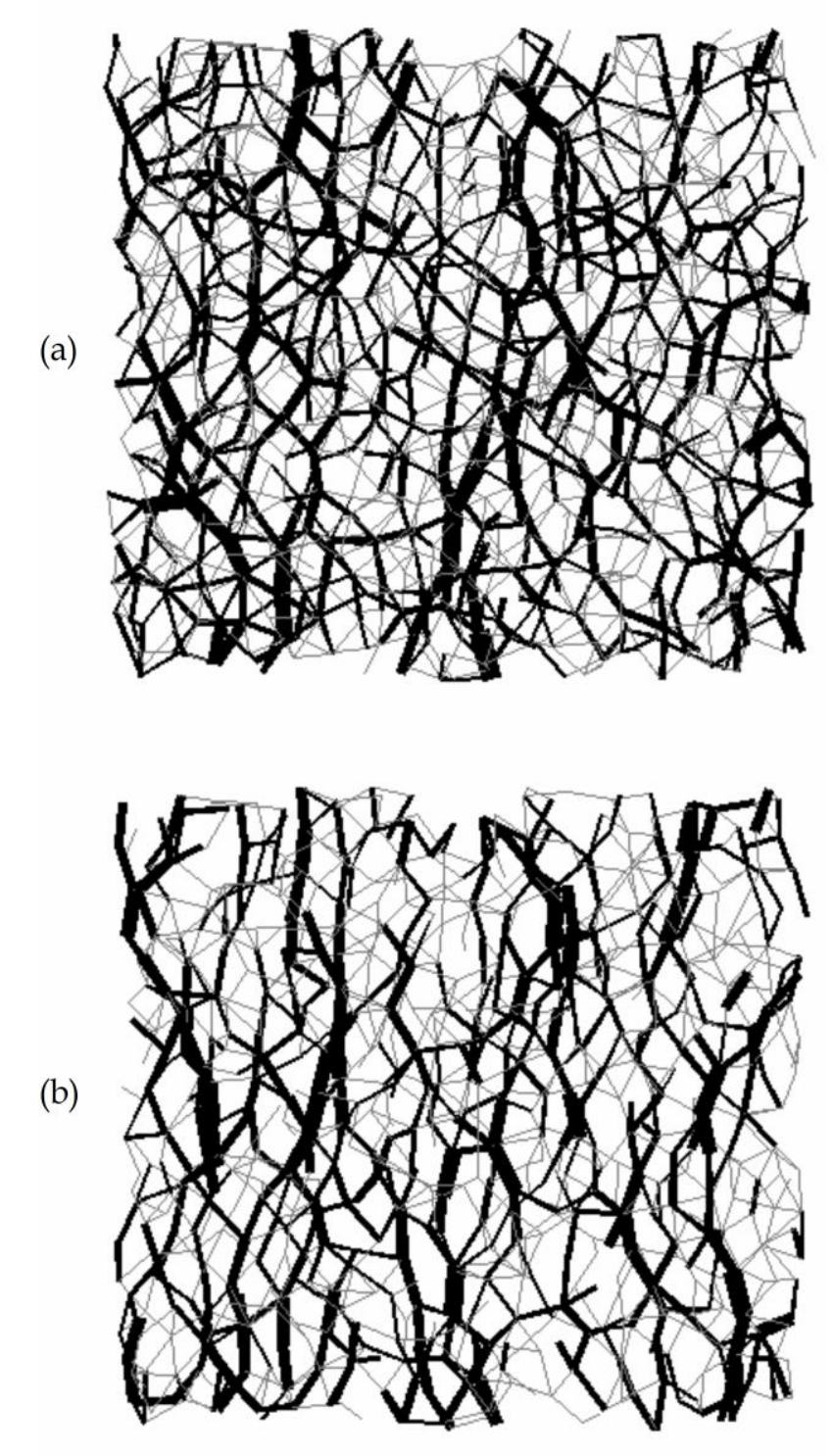

Figure 5 

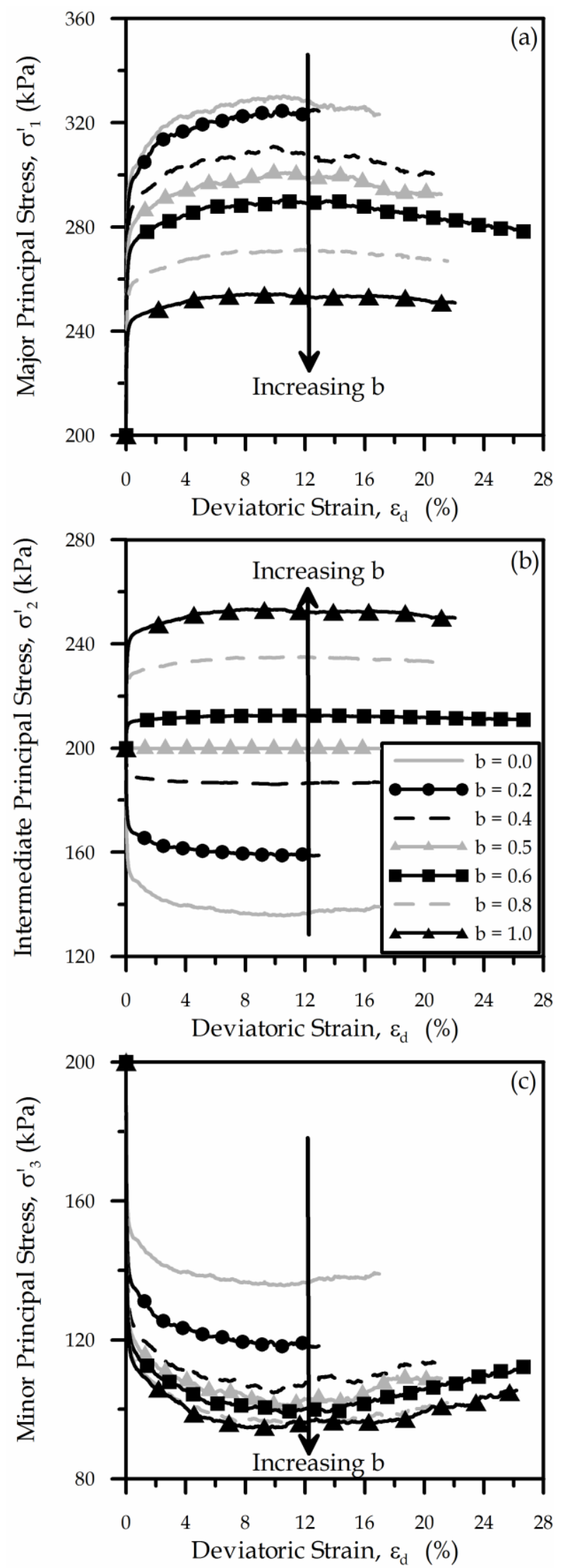

Figure 6 

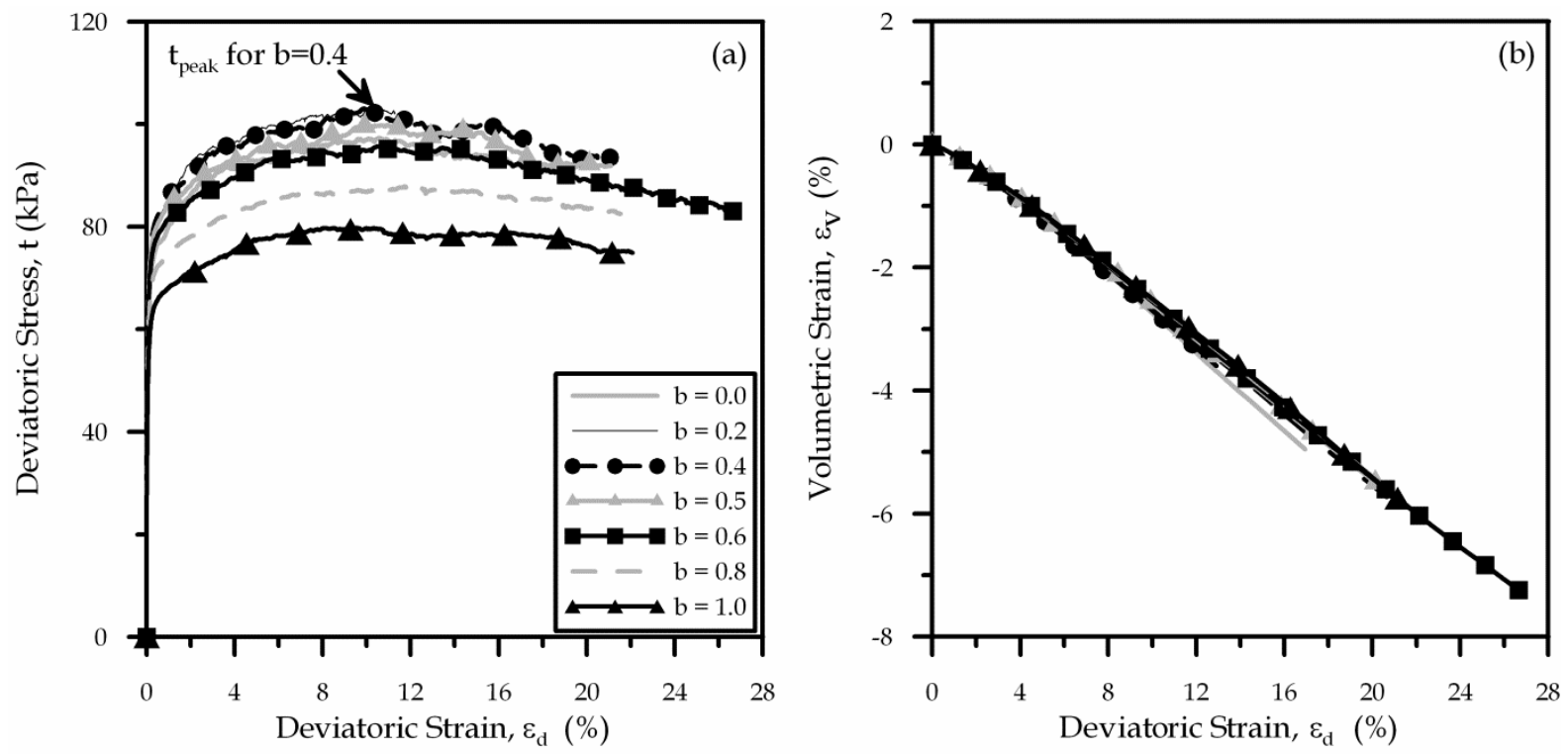

Figure 7 

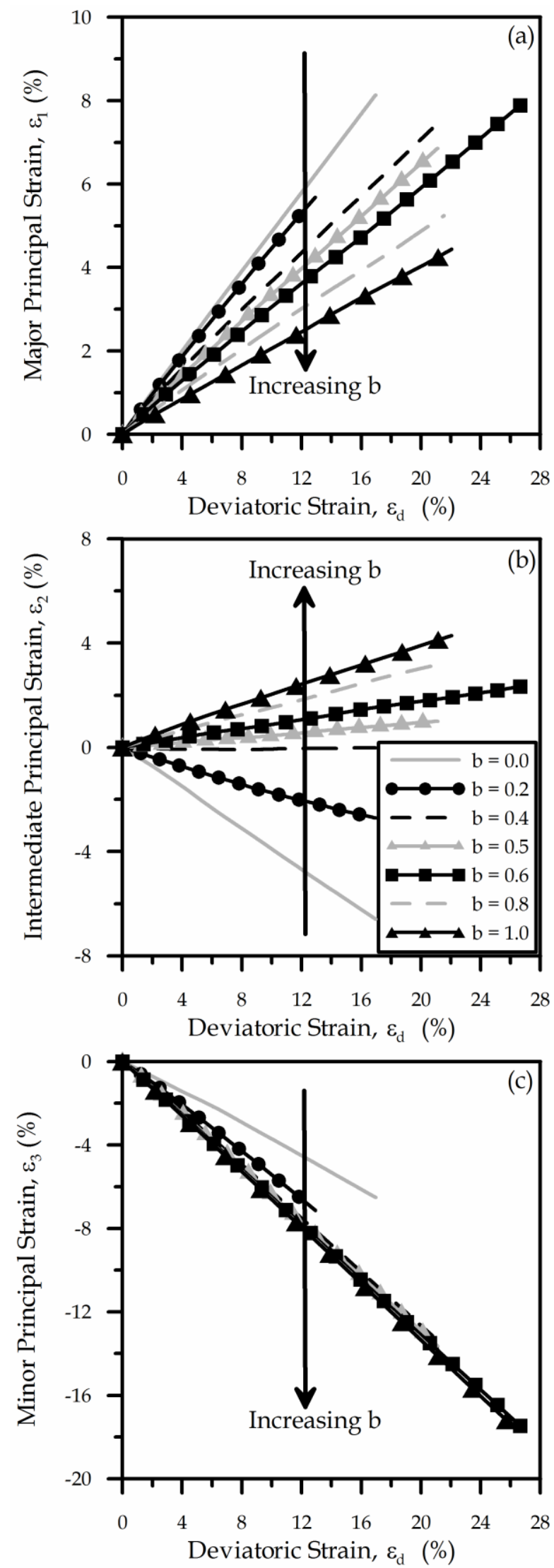

Figure 8 

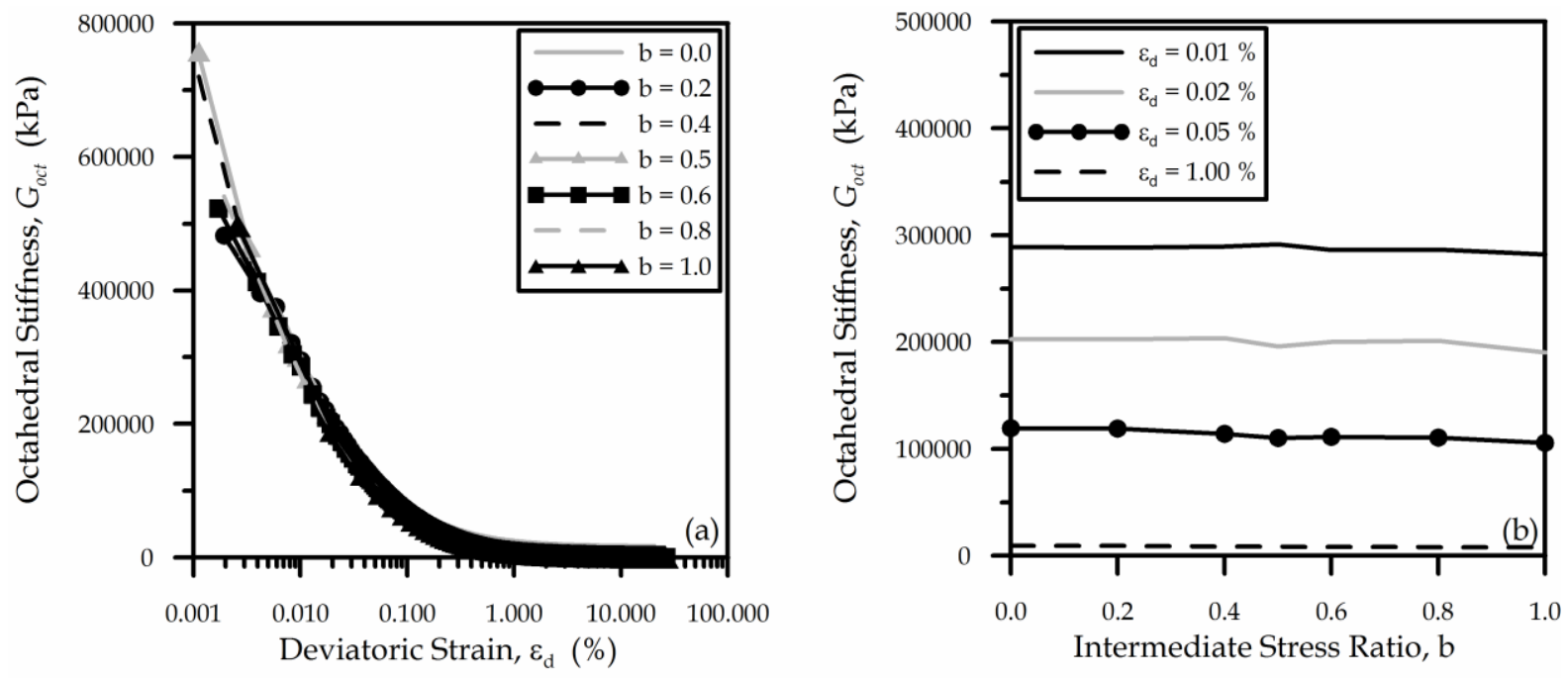

Figure 9 

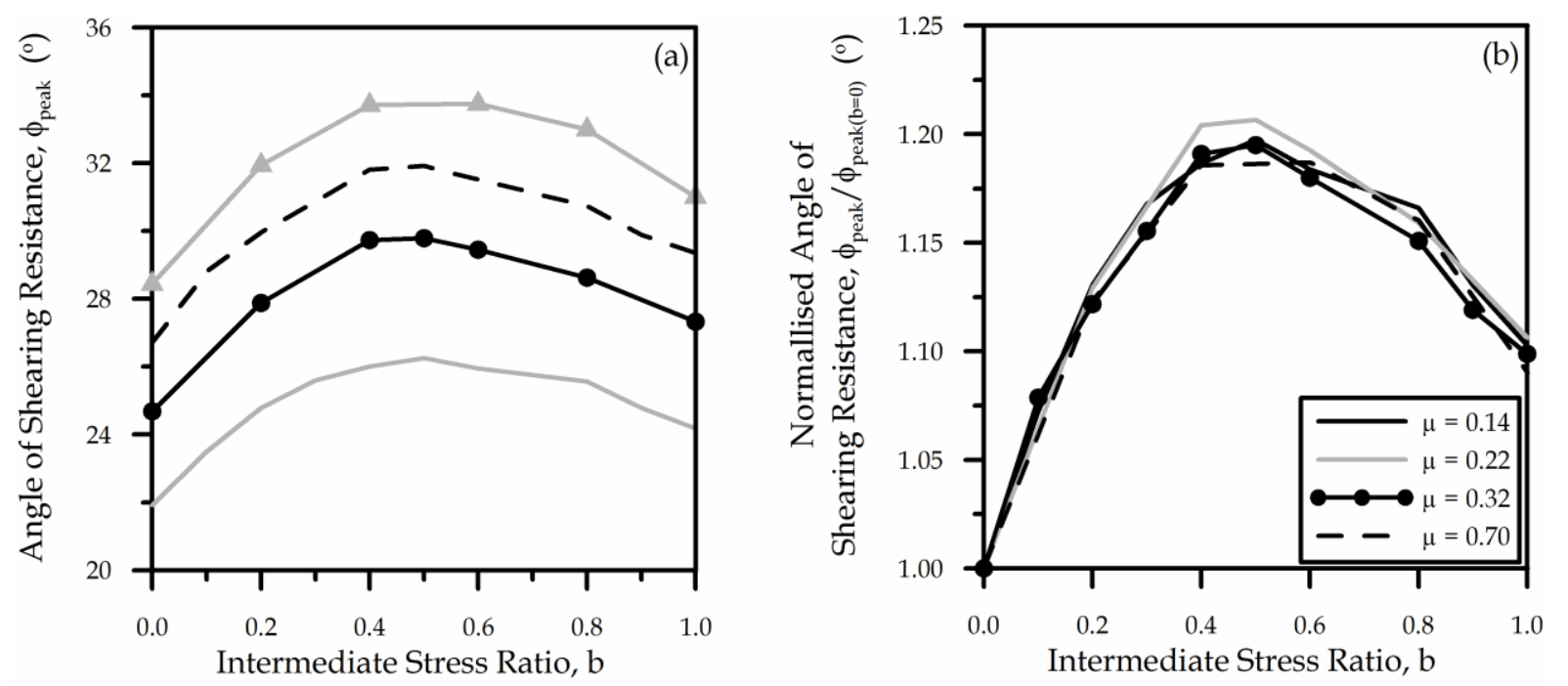

Figure 10 


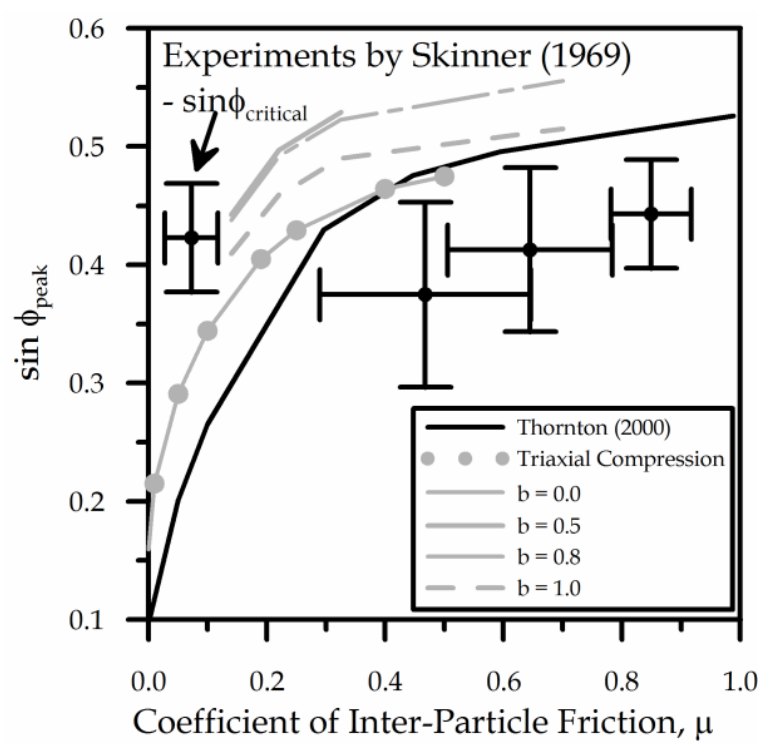

Figure 11 


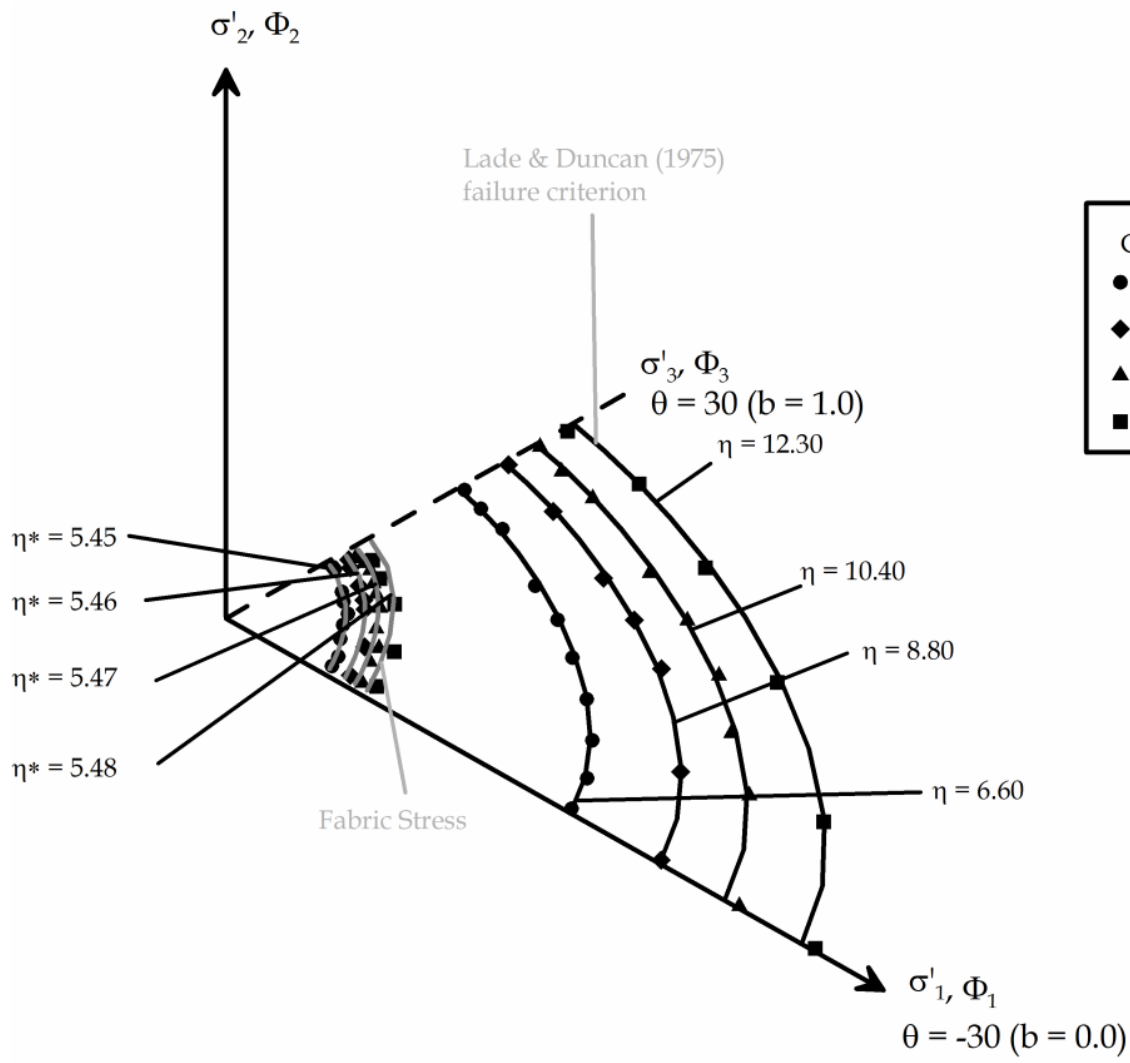

Figure 12 


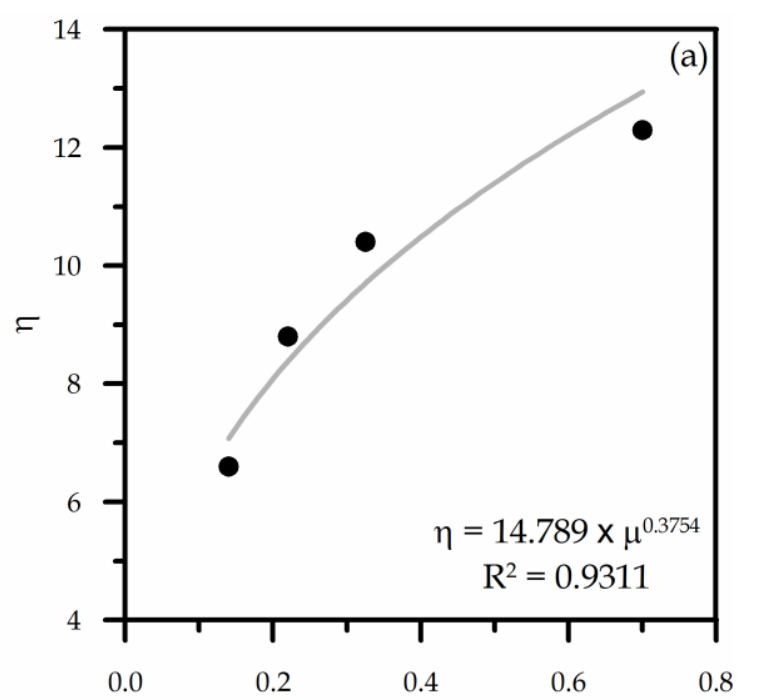

Coefficient of Inter-particle Friction, $\mu$

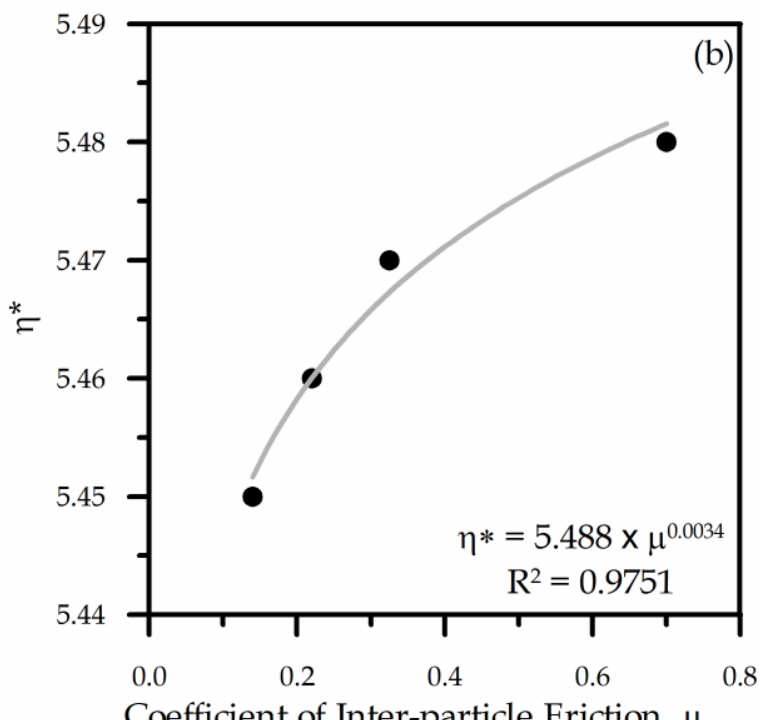

Coefficient of Inter-particle Friction, $\mu$

Figure 13 

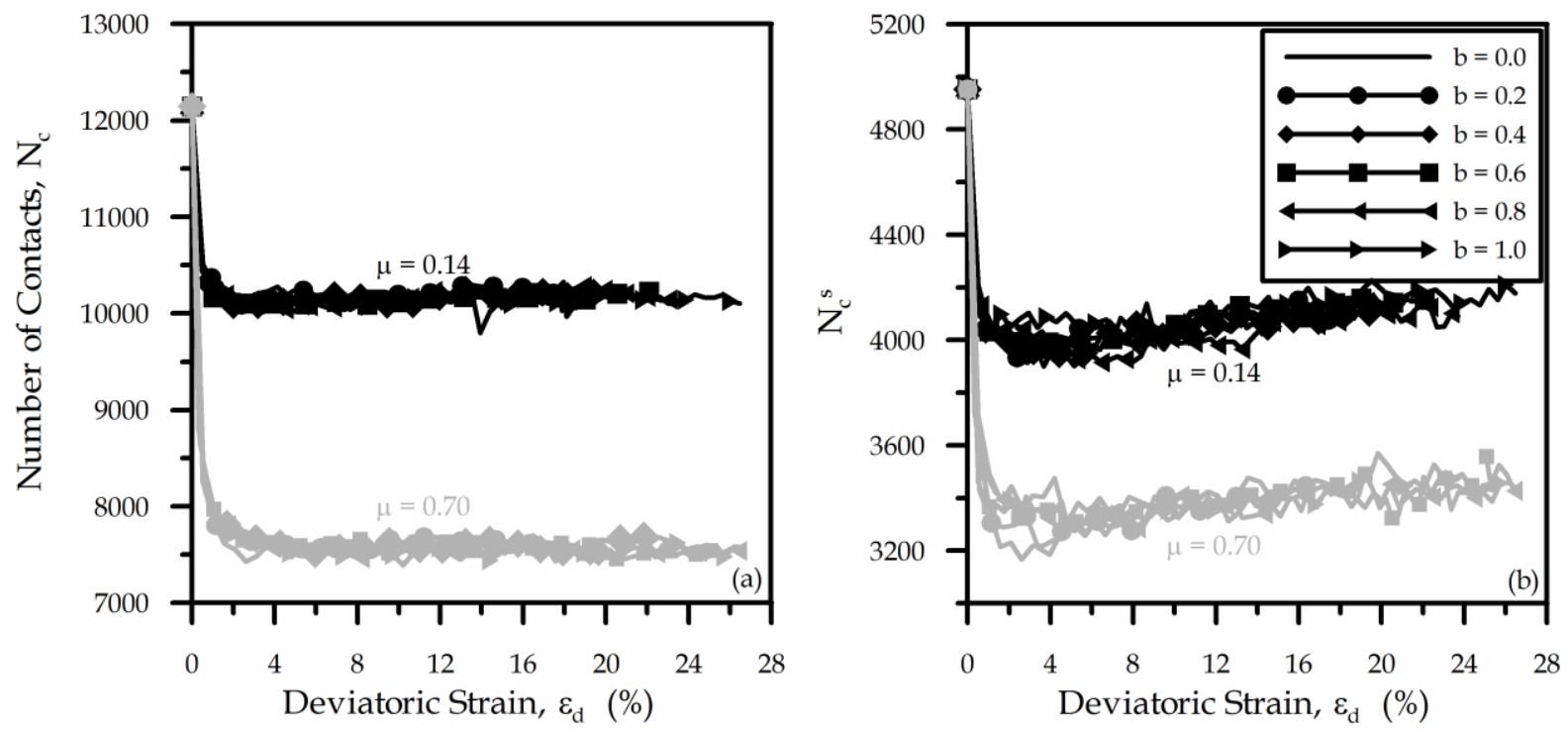

Figure 14 


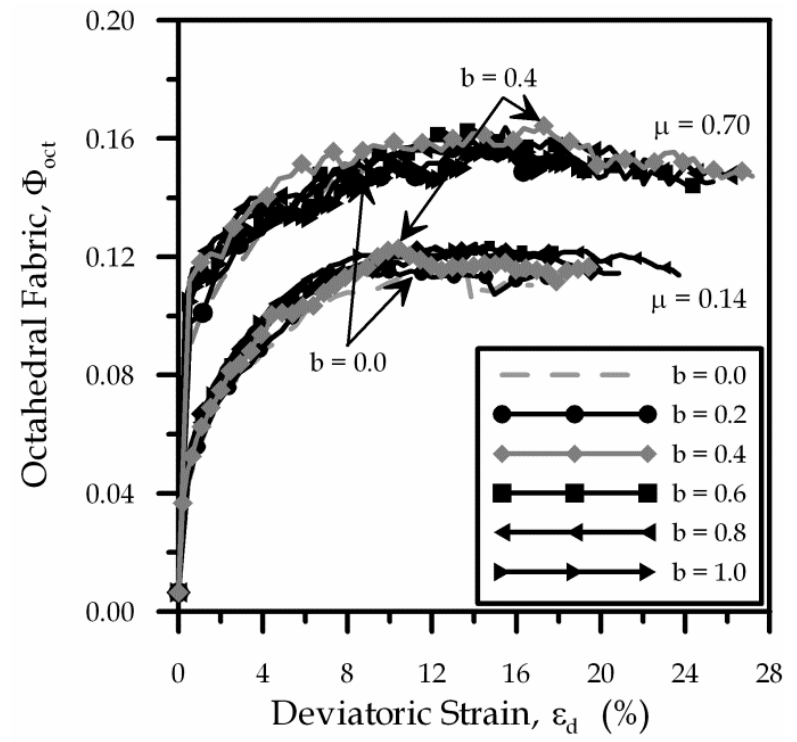

Figure 15 

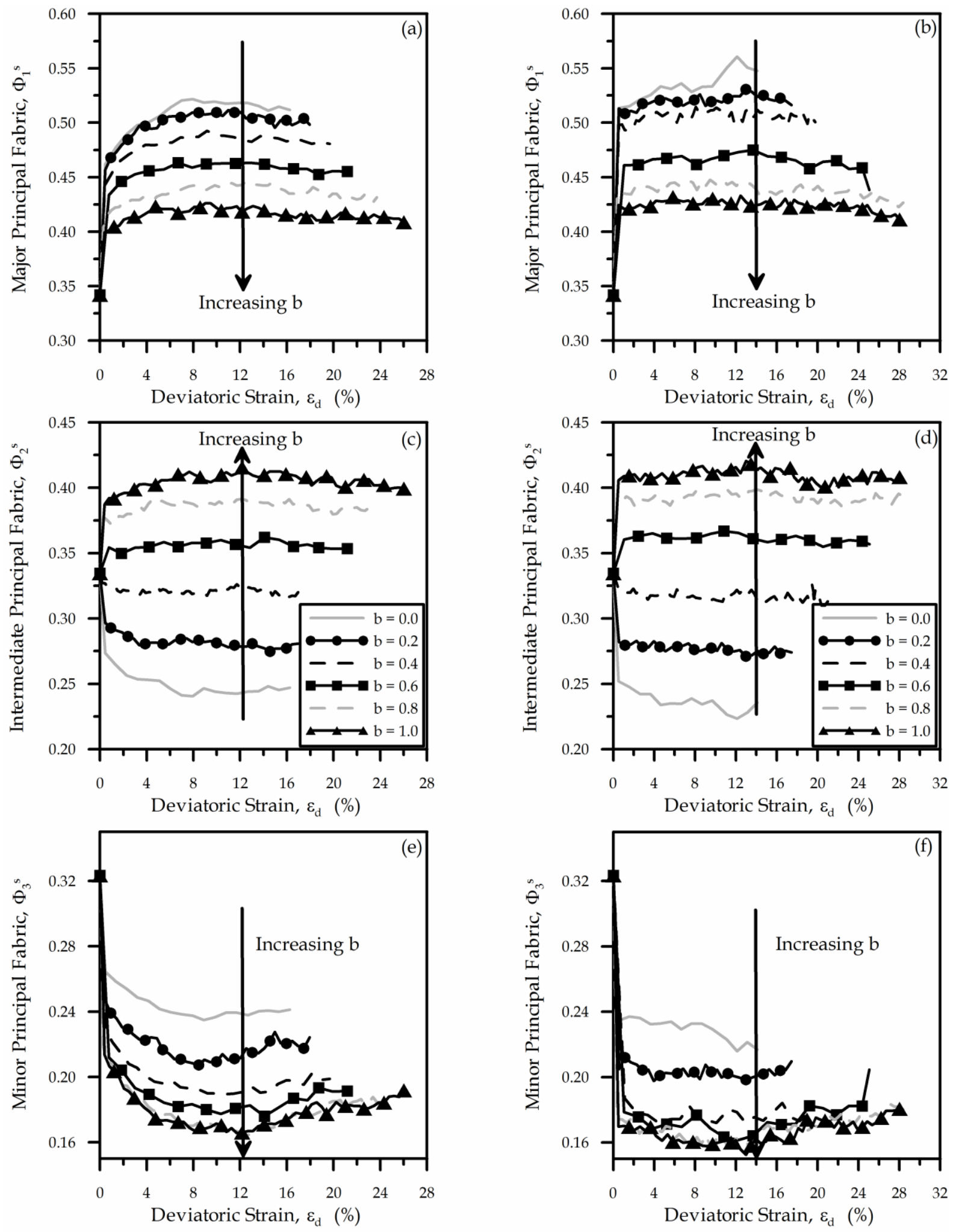

Figure 16 


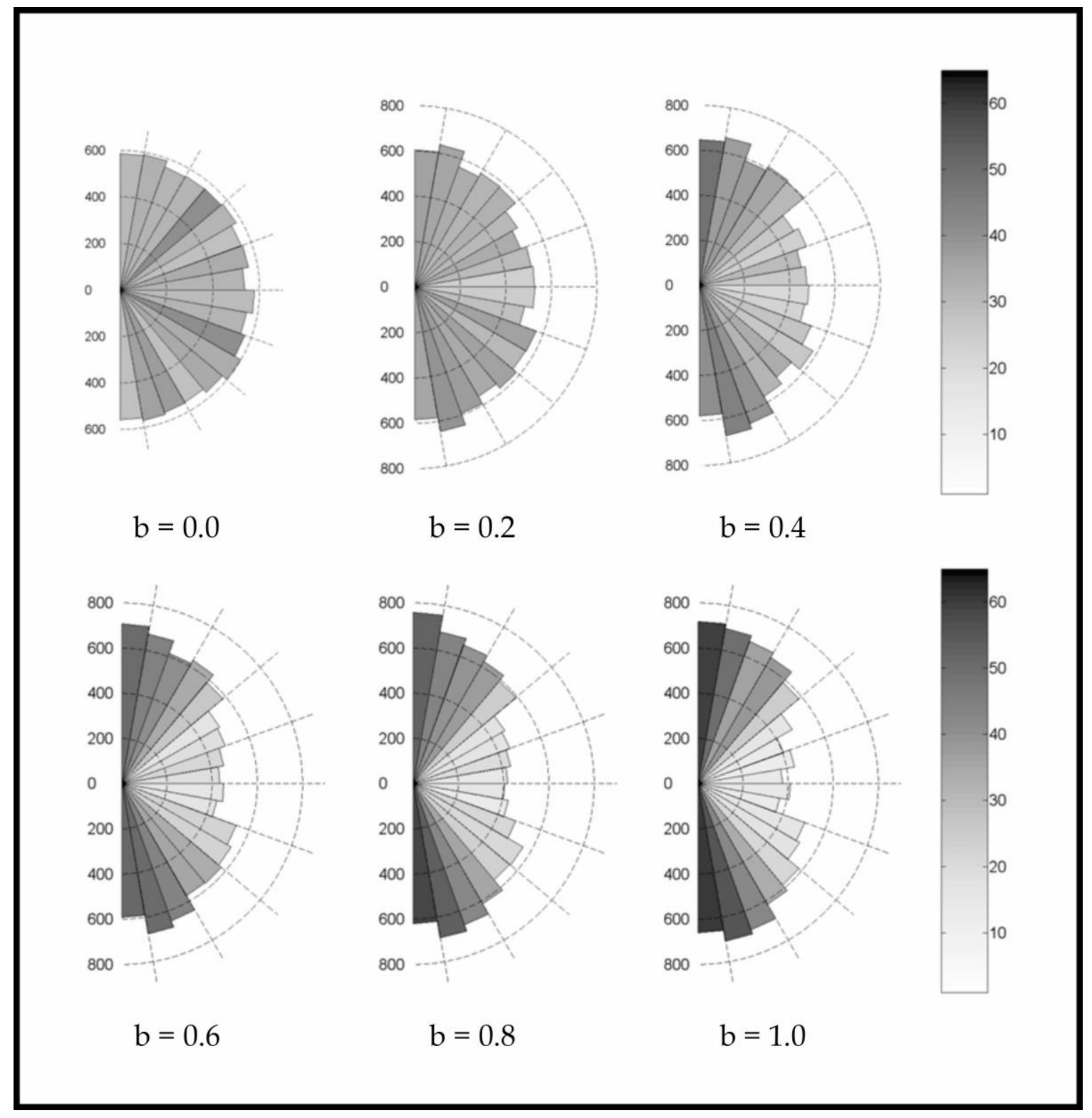

Figure 17 


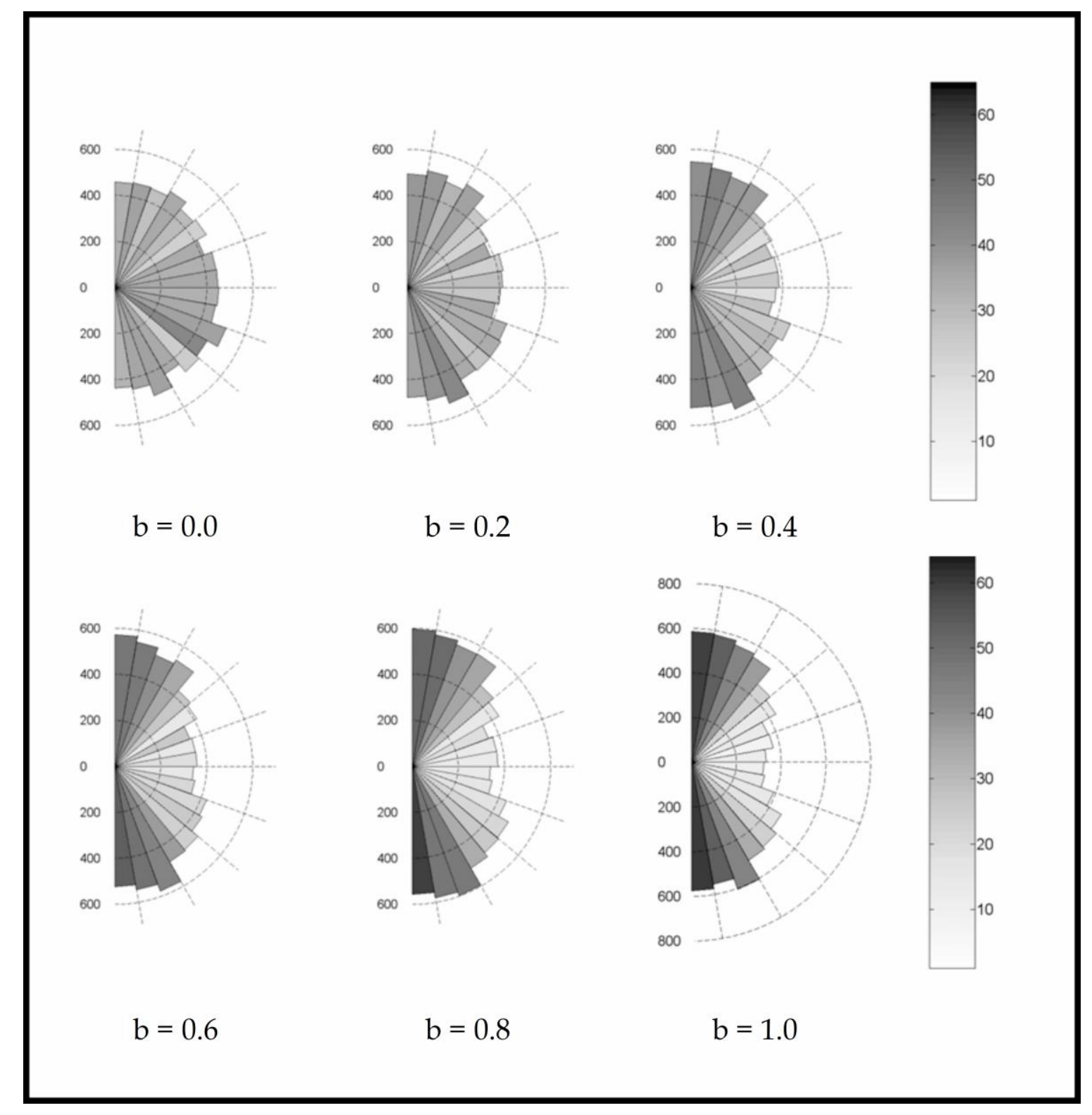

Figure 18 